\title{
Approximation simultanée par des produits de puissances de nombres algébriques
}

\author{
par \\ Michel Waldschmidt (Paris) \\ Dédié au Professeur J. W. S. Cassels \\ à l'occasion de ses 75 ans
}

1. Introduction. Soit $d$ un entier positif et soit $\Gamma$ un sous-groupe de type fini de $\left(\mathbb{C}^{\times}\right)^{d}$. L'adhérence de $\Gamma$ pour la topologie complexe est un groupe de Lie réel et on s'intéresse à l'approximation de ses points par des éléments de $\Gamma$. Dans ce texte on supposera que les coordonnées des éléments de $\Gamma$ sont des nombres algébriques : $\Gamma \subset\left(\overline{\mathbb{Q}}^{\times}\right)^{d}$, où $\overline{\mathbb{Q}}$ est la clôture algébrique de $\mathbb{Q}$ dans $\mathbb{C}$. En prenant l'image inverse par l'application exponentielle, on se ramène à l'étude de l'approximation des points d'un sous-espace vectoriel réel de $\mathbb{C}^{d}$ par des éléments d'un sous-groupe additif $Y$, formé de points dont les coordonnées sont des logarithmes de nombres algébriques : $Y \subset \mathcal{L}^{d}$, où $\mathcal{L}$ désigne le $\mathbb{Q}$-espace vectoriel formé des logarithmes de nombres algébriques:

$$
\mathcal{L}=\exp ^{-1}\left(\overline{\mathbb{Q}}^{\times}\right)=\left\{\lambda \in \mathbb{C}: e^{\lambda} \in \overline{\mathbb{Q}}^{\times}\right\} .
$$

Commençons par le cas réel. D'après un résultat de Kronecker (voir par exemple [3], [7], th. 442 , et [2], chap. $7, \S 1, \mathrm{n}^{\circ} 3$, corollaire 2 de la proposition 7 ), une condition nécessaire et suffisante pour qu'un sous-groupe de type fini $Y$ de $\mathbb{R}^{d}$ soit dense dans $\mathbb{R}^{d}$ est que, pour toute forme linéaire non nulle $\varphi: \mathbb{R}^{d} \rightarrow \mathbb{R}$, l'image $\varphi(Y)$ ait un rang $\geq 2$ sur $\mathbb{Z}$. Ainsi, en posant $V=\operatorname{Ker} \varphi$, pour vérifier que $Y$ est dense il faut majorer le rang sur $\mathbb{Z}$ de $Y \cap V$ pour tout hyperplan $V$ de $\mathbb{R}^{d}$.

Considérons maintenant un sous-groupe de type fini de $\mathbb{C}^{d}$. Un théorème de transcendance, le théorème du sous-groupe linéaire (cf. théorème 2.1 cidessous), permet de majorer la dimension du $\mathbb{Q}$-espace vectoriel $\mathcal{L}^{d} \cap V$ quand $V$ est un hyperplan de $\mathbb{C}^{d}$ dont l'intersection avec $\mathbb{Q}^{d}$ est réduite

L'auteur a bénéficié du soutien de la Japan Society for the Promotion of Science : bourse JSPS 96029. 
à $\{0\}$. Pour un hyperplan $V$ de $\mathbb{C}^{d}$ contenant un élément non nul de $\mathbb{Q}^{d}$, le $\mathbb{Q}$-espace vectoriel $\mathcal{L}^{d} \cap V$ est de dimension infinie. Aussi, $Y$ étant un sous-groupe de type fini de $\mathcal{L}^{d}$, pour pouvoir majorer le rang de $Y \cap V$ pour tout hyperplan $V$ de $\mathbb{C}^{d}$, on est amené à introduire une hypothèse : il faut supposer que $Y$ ne contient pas trop de points dans un même hyperplan rationnel sur $\mathbb{Q}$.

DÉfinition. Un sous-groupe de type fini $Y$ de $\mathbb{C}^{d}$ possède la propriété (IL) si, pour tout sous-espace $W$ de $\mathbb{C}^{d}$ rationnel sur $\mathbb{Q}$ de dimension $<d$, on a $Y \cap W=\{0\}$.

Si $y_{1}, \ldots, y_{l}$ est une base de $Y$ sur $\mathbb{Z}$, avec $y_{j}=\left(u_{1 j}, \ldots, u_{d j}\right)$, la propriété (IL) signifie que les coordonnées $u_{i j}$ des $y_{j}$ satisfont la condition d'indépendance linéaire suivante :

- pour tout $t=\left(t_{1}, \ldots, t_{d}\right) \in \mathbb{Z}^{d}, t \neq(0, \ldots, 0)$, et tout $s=\left(s_{1}, \ldots, s_{l}\right) \in$ $\mathbb{Z}^{l}, s \neq(0, \ldots, 0)$, on $a$

$$
\sum_{i=1}^{d} \sum_{j=1}^{l} t_{i} s_{j} u_{i j} \neq 0 .
$$

On pourrait affaiblir cette condition (IL), en imposant seulement une majoration au rang de $Y \cap W$ en fonction de la codimension de $W$ dans $\mathbb{C}^{d}$ (voir par exemple le $\S 1.3$ de [19]), mais cela alourdirait les énoncés. Nous donnerons seulement un exemple dans cette direction (corollaire 2.4).

Quand les nombres $u_{i j}$ sont tous réels, la propriété (IL) s'écrit de manière équivalente sous la forme multiplicative suivante, avec $\alpha_{i j}=e^{u_{i j}}$ :

- pour tout $t=\left(t_{1}, \ldots, t_{d}\right) \in \mathbb{Z}^{d}, t \neq(0, \ldots, 0)$, et tout $s=\left(s_{1}, \ldots, s_{l}\right) \in$ $\mathbb{Z}^{l}, s \neq(0, \ldots, 0)$, on $a$

$$
\prod_{i=1}^{d} \prod_{j=1}^{l} \alpha_{i j}^{t_{i} s_{j}} \neq 1 .
$$

Cela signifie que le sous-groupe $\Gamma$ de $\mathbb{G}_{\mathrm{m}}^{d}(\mathbb{R})=\left(\mathbb{R}^{\times}\right)^{d}$, engendré par $\gamma_{1}, \ldots$ $\ldots, \gamma_{l}$, avec $\gamma_{j}=\left(\alpha_{1 j}, \ldots, \alpha_{d j}\right)(1 \leq j \leq l)$, qui est de rang $l$, possède la propriété suivante :

- pour tout sous-groupe algébrique $G^{*}$ de $\mathbb{G}_{\mathrm{m}}^{d}$ de dimension $<d$, on a

$$
\Gamma \cap G^{*}(\mathbb{R})=\{1\} \text {. }
$$

Quand les nombres $u_{i j}$ ne sont pas tous réels, sous la condition (IL), on peut seulement majorer le rang de $\Gamma \cap G^{*}(\mathbb{C})$ quand $G^{*}$ est un sous-groupe algébrique de $\mathbb{G}_{\mathrm{m}}^{d}$ (cf. lemme 4.2).

La question de densité soulève, nous l'avons vu, un problème d'irrationalité. Nous considèrerons aussi le problème de transcendance qui lui est naturellement associé. Plus généralement, nous déduirons le résultat suivant 
d'une minoration (2.3) de la dimension du $\overline{\mathbb{Q}}$-espace vectoriel engendré $\operatorname{par} \varphi(Y)$.

ThÉORÈme 1.1. Soient $Y=\mathbb{Z} y_{1}+\ldots+\mathbb{Z} y_{l}$ un sous-groupe de $\mathcal{L}^{d}$ de rang l possédant la propriété (IL) et $\varphi: \mathbb{C}^{d} \rightarrow \mathbb{C}$ une forme linéaire non nulle.

(a) Si $l \geq d^{2}-d+1$, alors un au moins des $l$ nombres $\varphi\left(y_{j}\right)(1 \leq j \leq l)$ n'est pas nul.

(b) Si $l \geq d^{2}-d+2$, alors un au moins des $l$ nombres $\varphi\left(y_{j}\right)(1 \leq j \leq l)$ est irrationnel.

(c) Si $l \geq d^{2}+1$, alors un au moins des $l$ nombres $\varphi\left(y_{j}\right)(1 \leq j \leq l)$ est transcendant.

L'énoncé (a) s'écrit $\operatorname{rang}_{\mathbb{Z}}(Y \cap \operatorname{Ker} \varphi) \leq d^{2}-d$, ou encore $\operatorname{rang}_{\mathbb{Z}} \varphi(Y) \geq$ $l-d^{2}+d$. Il entraîne donc immédiatement (b). Cet énoncé d'irrationalité (b) a été utilisé par Damien Roy dans [14] pour démontrer le résultat suivant, répondant à un problème posé par Sansuc [15] :

- Soit $k$ un corps de nombres de degré $d=r_{1}+2 r_{2}$ sur $\mathbb{Q}$, où $r_{1}$ est le nombre de plongements de $k$ dans $\mathbb{R}$ et $2 r_{2}$ le nombre de plongements non réels deux-à-deux conjugués de $k$ dans $\mathbb{C}$. Il existe un sous-groupe de type fini de $k^{\times}$de rang $r_{1}+r_{2}+1$ dont l'image par le plongement canonique $k^{\times} \rightarrow\left(\mathbb{R}^{\times}\right)^{r_{1}} \times\left(\mathbb{C}^{\times}\right)^{r_{2}}$ est dense.

Le théorème principal de ce texte (théorème 1.6) représente une première étape vers un analogue quantitatif de ce résultat de densité de D. Roy.

Considérons maintenant un sous-groupe $Y=\mathbb{Z} y_{1}+\ldots+\mathbb{Z} y_{l}$ de type fini et de rang $l$ de $\mathbb{R}^{d}$. Choisissons une norme $|\cdot|$ sur $\mathbb{R}^{d}$. Si $Y$ est dense, pour chaque $\xi=\left(\xi_{1}, \ldots, \xi_{d}\right) \in \mathbb{R}^{d}$, la fonction

$$
T \mapsto \min _{\substack{t \in \mathbb{Z}^{l} \\\left|t_{j}\right| \leq T}}\left|\xi-t_{1} y_{1}-\ldots-t_{l} y_{l}\right|
$$

tend vers 0 en décroissant quand $T$ tend vers l'infini. La "vitesse" de décroissance de cette fonction donne une "mesure" de la densité de $Y$ dans $\mathbb{R}^{d}$.

Deux outils nous permettront d'établir une telle estimation. Le premier est un lemme de transfert (que nous allons exposer dans le paragraphe 3), qui permet de ramener la question de densité effective à une minoration de $\min _{1 \leq j \leq l}\left\|\varphi\left(y_{j}\right)\right\|$, quand $\|\cdot\|$ désigne la distance à l'entier le plus proche et $\varphi: \mathbb{C}^{d} \rightarrow \mathbb{C}$ est une forme linéaire non nulle. La minoration en question dépend du maximum des coefficients de $\varphi$ : pour $\varphi(z)=\vartheta_{1} z_{1}+\ldots+\vartheta_{d} z_{d}$, on posera

$$
\mathrm{N}(\varphi)=\max _{1 \leq i \leq d}\left|\vartheta_{i}\right|
$$


Le second outil est donc un énoncé d'approximation diophantienne, raffinement quantitatif du théorème 1.1 qui fournit les estimations requises pour l'utilisation du lemme de transfert. Pour énoncer ces estimations nous introduisons les notations suivantes.

- Soient $d, l$ deux entiers positifs et $Y=\mathbb{Z} y_{1}+\ldots+\mathbb{Z} y_{l}$ un sous-groupe de $\mathcal{L}^{d}$ de rang l possédant la propriété (IL). On écrit $y_{j}=\left(u_{1 j}, \ldots, u_{d j}\right)$ $(1 \leq j \leq l)$ et on pose $\alpha_{i j}=\exp \left(u_{i j}\right)(1 \leq i \leq d, 1 \leq j \leq l)$. Soit $K$ un corps de nombres de degré $D$ sur $\mathbb{Q}$ contenant les dl nombres $\alpha_{i j}$. On désigne par $A$ un nombre réel positif satisfaisant, pour $1 \leq i \leq d$ et $1 \leq j \leq l$,

$$
\log A \geq \max \left\{e / D, \mathrm{~h}\left(\alpha_{i j}\right),(e / D)\left|u_{i j}\right|\right\} .
$$

Enfin soit $\varphi: \mathbb{C}^{d} \rightarrow \mathbb{C}$ une forme linéaire non nulle.

La version quantitative suivante de la partie (a) du théorème 1.1 sera établie au paragraphe 4 :

ThÉORÈme 1.2. On suppose $d \geq 2$ et $l \geq d^{2}-d+1$. Alors

$$
\max _{1 \leq j \leq l}\left|\varphi\left(y_{j}\right)\right| \geq \mathrm{N}(\varphi) \exp \left\{-c_{0}(D \log A)^{\kappa_{0}}\right\}
$$

avec

$$
\kappa_{0}=\kappa_{0}(d, l)=\frac{d l}{l-d^{2}+d} \quad \text { et } \quad c_{0}=c_{0}(d, l)=d^{7 d^{3}} l^{d} .
$$

On en déduira (également au paragraphe 4) un raffinement quantitatif de la partie (b) du théorème 1.1 :

Corollaire 1.3. On pose $N=\max \{1, \mathrm{~N}(\varphi)\}$. Si $l \geq d^{2}-d+2$, alors

$$
\max _{1 \leq j \leq l}\left\|\varphi\left(y_{j}\right)\right\| \geq \mathrm{N}(\varphi) \exp \left\{-c_{1} N^{\kappa_{1}}\right\}
$$

avec

$$
\kappa_{1}=\frac{d(l-1)}{l-d^{2}+d-1} \quad \text { et } \quad c_{1}= \begin{cases}c_{0}(d, l-1) d^{\kappa_{1}}(D \log A)^{2 \kappa_{1}} & \text { si } d \geq 2, \\ D \log 2+(D \log A)^{2} & \text { si } d=1 .\end{cases}
$$

$\mathrm{Au}$ paragraphe 5 nous démontrerons la version effective suivante de la partie (c) du théorème 1.1 :

THÉORÈme 1.4. Supposons $d \geq 1$ et $l \geq d^{2}+1$. Soient $\beta_{1}, \ldots, \beta_{l}$ des éléments de $K$, non tous nuls, et soit $B \geq e / D$ un nombre réel tel que

$$
\log B \geq \max _{1 \leq j \leq l} \mathrm{~h}\left(\beta_{j}\right) .
$$

Alors

$$
\max _{1 \leq j \leq l}\left|\varphi\left(y_{j}\right)-\beta_{j}\right| \geq \exp \left\{-c_{2}(D \log A)^{d \kappa_{2}}(D \log B)^{\kappa_{2}}\right\},
$$

avec

$$
\kappa_{2}=\frac{l}{l-d^{2}} \quad \text { et } \quad c_{2}= \begin{cases}d^{17 d^{3}} l^{d} & \text { si } d \geq 2 \\ 10^{10}(l-1)^{2} & \text { si } d=1\end{cases}
$$


A titre de curiosité on déduira (au paragraphe 5) du cas particulier $d=1, l=2$ du théorème 1.4 une minoration d'une combinaison linéaire de deux logarithmes. Ce n'est pas la meilleure estimation connue (comparer notamment à [9]), mais elle est cependant déjà assez fine, vu le peu d'effort que nous avons consacré à cet aspect de la question.

Corollaire 1.5. Soient $\lambda_{1}$ et $\lambda_{2}$ deux éléments de $\mathcal{L}$ linéairement indépendants sur $\mathbb{Q}$ et soit $\beta$ un nombre algébrique. On pose $\alpha_{j}=e^{\lambda_{j}}(j=1,2)$, et on désigne par $D$ le degré du corps de nombres $\mathbb{Q}\left(\alpha_{1}, \alpha_{2}, \beta\right)$ sur $\mathbb{Q}$. Soient $A$ et $B$ deux nombres réels positifs satisfaisant

$A \geq \max \left\{e / D, \mathrm{~h}\left(\alpha_{1}\right), \mathrm{h}\left(\alpha_{2}\right),(e / D)\left|\lambda_{1}\right|,(e / D)\left|\lambda_{2}\right|\right\}, \quad B \geq \max \{e / D, \mathrm{~h}(\beta)\}$. Alors

$$
\left|\beta \lambda_{1}-\lambda_{2}\right| \geq \exp \left\{-10^{10} D^{4}(\log A)^{2}(\log B)^{2}\right\} .
$$

Voici maintenant le théorème principal de cet article, qui fournit un résultat effectif de densité concernant un groupe multiplicatif formé de points à coordonnées algébriques. On le déduira des théorèmes 1.2 et 1.4 dans le paragraphe 6 .

ThÉORÈme 1.6. Soient $r_{1}$ et $r_{2}$ des entiers $\geq 0$ avec $n=r_{1}+r_{2}>0$. On pose aussi $d=r_{1}+2 r_{2}$. Soit $\Gamma$ un sous-groupe de $\left(\mathbb{R}_{+}^{\times}\right)^{r_{1}} \times\left(\mathbb{C}^{\times}\right)^{r_{2}}$, engendré par des éléments $\left(\alpha_{i j}\right)_{1 \leq i \leq n}(j=1, \ldots, l)$. On suppose que pour tout $a=\left(a_{1}, \ldots, a_{d}\right) \in \mathbb{Z}^{d}, a \neq 0$, et tout $b=\left(b_{1}, \ldots, b_{l}\right) \in \mathbb{Z}^{l}, b \neq 0$, on $a$

$$
\prod_{j=1}^{l}\left(\prod_{i=1}^{n} \alpha_{i j}^{a_{i}} \prod_{k=r_{1}+1}^{n} \bar{\alpha}_{k j}^{a_{n+k}}\right)^{b_{j}} \neq 1 .
$$

(a) On suppose $l \geq d^{2}-d+2$, et on pose

$$
\theta_{1}=\frac{1}{d}-\frac{d-1}{l-1} \text {. }
$$

Il existe des constantes positives $c_{3}$ et $c_{4}$, ne dépendant que de $d, l$ et des $\alpha_{i j}$, possédant la propriété suivante : pour tout $\zeta=\left(\zeta_{1}, \ldots, \zeta_{n}\right) \in\left(\mathbb{R}_{+}^{\times}\right)^{r_{1}} \times$ $\left(\mathbb{C}^{\times}\right)^{r_{2}}$, et pour tout $T \geq c_{3} \max \left\{1,|\log | \zeta_{1}||, \ldots,|\log | \zeta_{n}||\right\}$, le système d'inéquations

$$
\max _{1 \leq j \leq l}\left|t_{j}\right| \leq T \quad \text { et } \quad \max _{1 \leq i \leq n}\left|\zeta_{i}-\alpha_{i 1}^{t_{1}} \ldots \alpha_{i l}^{t_{l}}\right| \leq c_{4}(\log T)^{-\theta_{1}}
$$

admet une solution $\left(t_{1}, \ldots, t_{l}\right) \in \mathbb{Z}^{l}$.

(b) On suppose $l>d^{2}$, et on pose

$$
\theta_{2}= \begin{cases}\left(l-d^{2}\right) / l & \text { si } d \geq 2, \\ 1 & \text { si } d=1 .\end{cases}
$$

Alors la même conclusion vaut avec la borne

$$
\max _{1 \leq i \leq n}\left|\zeta_{i}-\alpha_{i 1}^{t_{1}} \ldots \alpha_{i l}^{t_{l}}\right| \leq \exp \left\{-c_{5}(\log T)^{\theta_{2}}\right\},
$$

pour une constante $c_{5}$ qui ne dépend que de $d, l$ et des $\alpha_{i j}$. 
Re m a r que. La condition $l \geq d^{2}-d+2$ s'écrit aussi $\theta_{1}>0$. L'estimation obtenue en (a) est assez faible, puisqu'elle s'écrit

$$
\max _{1 \leq i \leq n}\left|\zeta_{i}-\alpha_{i 1}^{t_{1}} \ldots \alpha_{i l}^{t_{l}}\right| \leq \exp \left\{-\theta_{1} \log \log T+\log c_{4}\right\} .
$$

Nous verrons d'ailleurs que pour $d=1$ elle s'obtient à partir de l'argument de Liouville (alors que pour $d \geq 2$ elle n'est pas triviale). L'estimation obtenue en (b) est sensiblement meilleure : on ne peut pas espérer un exposant $\theta_{2}$ supérieur à 1 .

Il semble raisonnable de conjecturer que, sous les hypothèses du théorème 1.6 avec $l>d$, la conclusion peut être remplacée par

$$
\max _{1 \leq i \leq n}\left|\zeta_{i}-\alpha_{i 1}^{t_{1}} \ldots \alpha_{i l}^{t_{l}}\right| \leq c_{6} T^{1-(l / d)+\varepsilon}
$$

pour tout $\varepsilon>0$, avec une constante $c_{6}$ ne dépendant que de $d, l$, des $\alpha_{i j}$ et de $\varepsilon$. Un tel énoncé serait optimal.

2. Transcendance sur les tores complexes. Voici une variante (théorème 2.1 de [20]) du théorème du sous-groupe linéaire. On en trouvera d'autres notamment dans [13]; voir aussi [4] et [21].

ThÉORÈme 2.1 (théorème du sous-groupe linéaire). Soient $d_{0}$ et $d_{1}$ deux entiers $\geq 0$ avec $d_{0}+d_{1}>0$. Soit $\mathcal{V}$ un sous-espace vectoriel de $\mathbb{C}^{d_{0}+d_{1}}$ tel que

$$
\mathcal{V} \cap\left(\overline{\mathbb{Q}}^{d_{0}} \times\{0\}^{d_{1}}\right)=\{0\} \quad \text { et } \quad \mathcal{V} \cap\left(\{0\}^{d_{0}} \times \mathbb{Q}^{d_{1}}\right)=\{0\} .
$$

Alors le $\mathbb{Q}$-espace vectoriel $\mathcal{V} \cap\left(\overline{\mathbb{Q}}^{d_{0}} \times \mathcal{L}^{d_{1}}\right)$ est de dimension finie majorée par

$$
\operatorname{dim}_{\mathbb{Q}}\left(\mathcal{V} \cap\left(\overline{\mathbb{Q}}^{d_{0}} \times \mathcal{L}^{d_{1}}\right)\right) \leq d_{1}\left(d_{0}+d_{1}-1\right) .
$$

Le corollaire suivant nous permettra d'établir le théorème 1.1.

Corollaire 2.2. Soit $Y$ un sous-groupe de type fini de $\mathcal{L}^{d}$ et soit $\varphi$ : $\mathbb{C}^{d} \rightarrow \mathbb{C}$ une forme linéaire non nulle. On désigne par $r$ la dimension $d u \overline{\mathbb{Q}}$ espace vectoriel engendré par $\varphi(Y)$. Alors il existe un sous-espace vectoriel $W$ de $\mathbb{C}^{d}$, de codimension $d_{1} \geq 1$, rationnel sur $\mathbb{Q}$, tel que

$$
\operatorname{rang}_{\mathbb{Z}}(Y / Y \cap W) \leq d_{1}\left(d_{1}+r-1\right) .
$$

Démonstration. Soit $W$ le sous-espace vectoriel de $\mathbb{C}^{d}$ sur $\mathbb{C}$ engendré par $\mathbb{Q}^{d} \cap \operatorname{Ker} \varphi$. C'est le plus grand sous-espace de $\mathbb{C}^{d}$, rationnel $\operatorname{sur} \mathbb{Q}$, qui soit contenu dans $\operatorname{Ker} \varphi$. On note $d_{1}$ la codimension de $W$ dans $\mathbb{C}^{d}$ et on choisit $d_{1}$ éléments de $\mathbb{Q}^{d}$ linéairement indépendants modulo $W$. Leurs images par la surjection canonique $\mathbb{C}^{d} \rightarrow \mathbb{C}^{d} / W$ donnent une base de $\mathbb{C}^{d} / W$, ce qui fournit une surjection $\pi: \mathbb{C}^{d} \rightarrow \mathbb{C}^{d_{1}}$ de noyau $W$ vérifiant $\pi\left(\mathbb{Q}^{d}\right)=\mathbb{Q}^{d_{1}}$. Soit $\widetilde{\varphi}: \mathbb{C}^{d_{1}} \rightarrow \mathbb{C}$ la forme linéaire déduite par passage au 
quotient de $\varphi$. Comme $W$ a été choisi maximal, le noyau $\operatorname{Ker} \widetilde{\varphi}=\pi(\operatorname{Ker} \varphi)$ de $\widetilde{\varphi}$ ne contient pas d'élément non nul de $\mathbb{Q}^{d_{1}}$. On écrit

$$
\widetilde{\varphi}(z)=\vartheta_{1} z_{1}+\ldots+\vartheta_{d_{1}} z_{d_{1}}, \quad z=\left(z_{1}, \ldots, z_{d_{1}}\right) \in \mathbb{C}^{d_{1}} .
$$

D'autre part, on choisit une base $\xi_{1}, \ldots, \xi_{r}$ du $\overline{\mathbb{Q}}$-espace vectoriel engendré par $\varphi(Y)$. On désigne d'abord par $\psi$ l'homomorphisme de groupes additifs de $Y$ dans $\overline{\mathbb{Q}}^{r}$ qui envoie $y \in Y$ sur les composantes de $\varphi(y)$ dans la base $\xi_{1}, \ldots, \xi_{r}$. On désigne ensuite par $\tilde{Y}$ l'image de $Y$ dans $\mathbb{C}^{r+d_{1}}$ par l'application $y \mapsto(\psi(y), \pi(y))$. On désigne enfin par $\mathcal{V}$ l'hyperplan de $\mathbb{C}^{r+d_{1}}$ d'équation

$$
\xi_{1} z_{1}+\ldots+\xi_{r} z_{r}=\vartheta_{1} z_{r+1}+\ldots+\vartheta_{d_{1}} z_{r+d_{1}} .
$$

On a $\mathcal{V} \cap\left(\overline{\mathbb{Q}}^{r} \times\{0\}^{d_{1}}\right)=\{0\}$ car $\xi_{1}, \ldots, \xi_{r}$ sont linéairement indépendants sur $\overline{\mathbb{Q}}$, et $\mathcal{V} \cap\left(\{0\}^{r} \times \mathbb{Q}^{d_{1}}\right)=\{0\}$ car $\mathcal{V} \cap\left(\{0\}^{r} \times \mathbb{C}^{d_{1}}\right)=\{0\}^{r} \times \operatorname{Ker} \widetilde{\varphi}$. Par conséquent, on peut appliquer le théorème 2.1 avec $d_{0}=r$ :

$$
\operatorname{dim}_{\mathbb{Q}}\left(\mathcal{V} \cap\left(\overline{\mathbb{Q}}^{r} \times \mathcal{L}^{d_{1}}\right)\right) \leq d_{1}\left(d_{1}+r-1\right) .
$$

On remarque pour terminer que $\tilde{Y}$ est un sous-groupe de $\mathbb{C}^{r+d_{1}}$ qui satisfait

$$
\operatorname{rang}_{\mathbb{Z}} \tilde{Y} \geq \operatorname{rang}_{\mathbb{Z}} \pi(Y) \quad \text { et } \quad \tilde{Y} \subset \mathcal{V} \cap\left(\overline{\mathbb{Q}}^{r} \times \mathcal{L}^{d_{1}}\right)
$$

donc

$$
\operatorname{rang}_{\mathbb{Z}}(Y / Y \cap W) \leq \operatorname{rang}_{\mathbb{Z}} \tilde{Y} \leq \operatorname{dim}_{\mathbb{Q}}\left(\mathcal{V} \cap\left(\overline{\mathbb{Q}}^{r} \times \mathcal{L}^{d_{1}}\right)\right) \leq d_{1}\left(d_{1}+r-1\right)
$$

Démonstration du théorème 1.1. Sous les hypothèses du corollaire 2.2, si le sous-groupe $Y$ possède la propriété (IL), alors son rang $l$ est borné par

$$
l \leq d(d+r-1) .
$$

On obtient la partie (a) du théorème 1.1 en prenant $r=0$ et la partie (c) en prenant $r=1$.

Remarque. De la partie (a) du théorème 1.1 on déduit le théorème des six exponentielles de Lang et Ramachandra ([8], chap. II, $\S 1$, th. 1 et [10], p. 67). De même le théorème de Gel'fond-Schneider ([6], chap. III, $\S 2$ et [17], chap. II, th. 14) résulte de la partie (c) du théorème 1.1. En prenant $d=1$ dans (2.3) on obtient la version homogène du théorème de Baker [1], chap. 2, th. 2.1 : des éléments $\mathbb{Q}$-linéairement indépendants de $\mathcal{L}$ sont $\overline{\mathbb{Q}}$-linéairement indépendants. Pour obtenir la version non homogène de ce même théorème : toute combinaison linéaire non nulle d'éléments de $\mathcal{L}$ à coefficients algébriques est transcendante, il faut faire intervenir des dérivations, ce que permet la version plus générale du théorème du sousgroupe linéaire (théorème 4.1 de [21], ou bien les variantes de [13]). A ce propos, on peut donner une démonstration "duale" du corollaire 2.2, utilisant le groupe algébrique $\mathbb{G}_{\mathrm{m}}^{l}$, avec $l=\operatorname{rang}_{\mathbb{Z}} Y$, où on remplace les facteurs 
$\mathbb{G}_{\mathrm{a}}^{r}$ par $r$ dérivations. Pour cela on considère, dans l'espace tangent $\mathbb{C}^{l}$, le sous-groupe de rang $d$ engendré par les vecteurs lignes de la matrice dont les vecteurs colonnes sont les composantes d'une base de $Y$ sur $\mathbb{Z}$. On considère aussi le sous-espace vectoriel de $\mathbb{C}^{l}$, rationnel sur $\overline{\mathbb{Q}}$, de dimension $r$, engendré par les vecteurs lignes de la matrice dont les colonnes sont les composantes de $\xi_{1}, \ldots, \xi_{r}$. Quand on se restreint à la situation du corollaire 1.5, cette démonstration duale s'apparente à la méthode de Gel'fond, tandis que celle que nous avons donnée est plus proche du point de vue de Schneider dans sa solution du septième problème de Hilbert.

En utilisant le théorème de Kronecker cité dans le premier paragraphe, on déduit du corollaire 2.2 le résultat de densité suivant :

Corollaire 2.4. Soit $Y$ un sous-groupe de $(\mathcal{L} \cap \mathbb{R})^{d}$. On suppose que pour tout entier $d_{1} \geq 1$ et tout sous-espace vectoriel $W$ de $\mathbb{R}^{d}$ rationnel sur $\mathbb{Q}$ de codimension $d_{1}$, on a

$$
\operatorname{rang}_{\mathbb{Z}}(Y / Y \cap W) \geq d_{1}\left(d_{1}-1\right)+2 .
$$

Alors $Y$ est dense dans $\mathbb{R}^{d}$.

Dans ce corollaire 2.4, la condition sur le rang de $Y / Y \cap W$ n'est probablement pas optimale, mais elle est naturelle : actuellement les méthodes de transcendance ne permettent pas d'établir la densité dans $\mathbb{R}^{d}$ d'un sousgroupe contenu dans $(\mathcal{L} \cap \mathbb{R})^{d}$ de rang $<d(d-1)+2$, même s'il possède la propriété (IL).

Démonstration. Soit $Y$ un sous-groupe de $(\mathcal{L} \cap \mathbb{R})^{d}$ qui n'est pas dense. D'après le théorème de Kronecker, il existe une forme linéaire non nulle $\varphi: \mathbb{R}^{d} \rightarrow \mathbb{R}$ telle que $\varphi(Y)$ soit de rang $<2$ sur $\mathbb{Z}$. Si $l$ est le rang de $Y$, alors celui $l^{\prime}$ du sous-groupe $Y^{\prime}=Y \cap \operatorname{Ker} \varphi$ vérifie $l^{\prime}>l-2$. Le corollaire 2.2 avec $r=0$ donne l'existence d'un sous-espace vectoriel $W$ de $\mathbb{C}^{d}$, de codimension $d_{1} \geq 1$, rationnel sur $\mathbb{Q}$, tel que

$$
\operatorname{rang}_{\mathbb{Z}}\left(Y^{\prime} / Y^{\prime} \cap W\right) \leq d_{1}\left(d_{1}-1\right) .
$$

Alors on a

$$
\operatorname{rang}_{\mathbb{Z}}(Y \cap W) \geq \operatorname{rang}_{\mathbb{Z}}\left(Y^{\prime} \cap W\right) \geq l^{\prime}-d_{1}\left(d_{1}-1\right)>l-d_{1}\left(d_{1}-1\right)-2,
$$

donc

$$
\operatorname{rang}_{\mathbb{Z}}(Y / Y \cap W)<d_{1}\left(d_{1}-1\right)-2
$$

\section{Lemme de transfert}

(a) Une version quantitative du théorème de Kronecker. On va utiliser une version quantitative d'un théorème de Kronecker. Rappelons déjà la version qualitative — voir [3]; voir aussi [23], chap. II, §4, ainsi que les travaux de D. Roy [11] et [12] sur les sous-groupes minimaux. Soient $m$ et 
$n$ deux entiers positifs, et soient $\vartheta_{j i}(1 \leq j \leq n, 1 \leq i \leq m)$ des nombres réels. On pose

$$
\gamma_{i}=\left(\vartheta_{1 i}, \ldots, \vartheta_{n i}\right) \in \mathbb{R}^{n} \quad(1 \leq i \leq m)
$$

et

$$
\delta_{j}=\left(\vartheta_{j 1}, \ldots, \vartheta_{j m}\right) \in \mathbb{R}^{m} \quad(1 \leq j \leq n) .
$$

Ainsi

$$
\Gamma=\mathbb{Z}^{n}+\mathbb{Z} \gamma_{1}+\ldots+\mathbb{Z} \gamma_{m} \subset \mathbb{R}^{n} \quad \text { et } \quad \Delta=\mathbb{Z}^{m}+\mathbb{Z} \delta_{1}+\ldots+\mathbb{Z} \delta_{n} \subset \mathbb{R}^{m}
$$

sont les sous-groupes engendrés par les vecteurs colonnes des matrices

$$
\left(\begin{array}{cccccc}
1 & \ldots & 0 & \vartheta_{11} & \ldots & \vartheta_{1 m} \\
\vdots & \ddots & \vdots & \vdots & \ddots & \vdots \\
0 & \ldots & 1 & \vartheta_{n 1} & \ldots & \vartheta_{n m}
\end{array}\right) \text { et }\left(\begin{array}{cccccc}
1 & \ldots & 0 & \vartheta_{11} & \ldots & \vartheta_{n 1} \\
\vdots & \ddots & \vdots & \vdots & \ddots & \vdots \\
0 & \ldots & 1 & \vartheta_{1 m} & \ldots & \vartheta_{n m}
\end{array}\right) \text {. }
$$

D'après Kronecker, $\Gamma$ est dense dans $\mathbb{R}^{n}$ si et seulement si $\Delta$ est de rang $n+m$ sur $\mathbb{Z}$. Un lemme de transfert de Khinchine permet de préciser ce résultat de la manière suivante (comparer avec le lemme 5.1 de [18]).

Lemme 3.1. Soient $\vartheta_{j i}(1 \leq j \leq n, 1 \leq i \leq m)$ des nombres réels, $T$ et $S$ des nombres réels positifs avec $T>1$.

(i) On pose $\eta=2^{-n-m}((n+m) !)^{2}$ et on suppose que pour tout $\left(s_{1}, \ldots, s_{n}\right)$ $\in \mathbb{Z}^{n} \backslash\{0\}$ vérifiant $\max _{1 \leq j \leq n}\left|s_{j}\right| \leq S$, on a

$$
\left\|s_{1} \delta_{1}+\ldots+s_{n} \delta_{n}\right\| \geq \eta T^{-1} .
$$

Alors pour tout $\zeta \in \mathbb{R}^{n}$, il existe $\left(t_{1}, \ldots, t_{m}\right) \in \mathbb{Z}^{m}$ vérifiant $\max _{1 \leq i \leq m}\left|t_{i}\right|$ $\leq T$, et tel que

$$
\left\|\zeta-t_{1} \gamma_{1}-\ldots-t_{m} \gamma_{m}\right\| \leq \eta S^{-1} .
$$

(ii) On suppose que pour tout $\zeta \in \mathbb{R}^{n}$, il existe $\left(t_{1}, \ldots, t_{m}\right) \in \mathbb{Z}^{m}$ vérifiant $\max _{1 \leq i \leq m}\left|t_{i}\right| \leq T$, et

$$
\left\|\zeta-t_{1} \gamma_{1}-\ldots-t_{m} \gamma_{m}\right\| \leq \frac{1}{2(n+m) S} .
$$

Alors pour tout $\left(s_{1}, \ldots, s_{n}\right) \in \mathbb{Z}^{n} \backslash\{0\}$ vérifiant $\max _{1 \leq j \leq n}\left|s_{j}\right| \leq S$, on a

$$
\left\|s_{1} \delta_{1}+\ldots+s_{n} \delta_{n}\right\| \geq \frac{1}{2(n+m) T} .
$$

On a noté $\|\cdot\|$ :

- la distance à $\mathbb{Z}^{m}$ dans l'hypothèse de (i) et dans la conclusion de (ii),

- la distance à $\mathbb{Z}^{n}$ dans l'hypothèse de (ii) et dans la conclusion de (i).

C'est surtout la partie (i) qui nous sera utile : elle ramène la question de densité effective à un problème d'approximation diophantienne homogène. La partie (ii) montre qu'il y a en fait équivalence entre les deux questions, à des constantes explicites près. 
La démonstration du lemme 3.1 utilise un lemme de transfert de Khinchine pour les formes linéaires $L_{1}, \ldots, L_{n}, M_{1}, \ldots, M_{m}$ définies par

$$
L_{j}(x)=\sum_{i=1}^{m} \vartheta_{j i} x_{i} \quad(1 \leq j \leq n) \quad \text { et } \quad M_{i}(u)=\sum_{j=1}^{n} \vartheta_{j i} u_{j} \quad(1 \leq i \leq m) .
$$

Lemme 3.2. Soient $C>0$ et $X>1$ des nombres réels. Soit $\zeta=$ $\left(\zeta_{1}, \ldots, \zeta_{n}\right) \in \mathbb{R}^{n}$.

(A) Une condition nécessaire pour qu'il existe $t \in \mathbb{Z}^{m}$ vérifiant

$$
\max _{1 \leq j \leq n}\left\|L_{j}(t)-\zeta_{j}\right\| \leq C \quad \text { et } \quad \max _{1 \leq i \leq m}\left|t_{i}\right| \leq X
$$

est que l'inégalité

$$
\left\|s_{1} \zeta_{1}+\ldots+s_{n} \zeta_{n}\right\| \leq \gamma \max \left\{X \max _{1 \leq i \leq m}\left\|M_{i}(s)\right\| ; C \max _{1 \leq j \leq n}\left|s_{j}\right|\right\}
$$

soit satisfaite pour tout $s \in \mathbb{Z}^{n}$ avec $\gamma=m+n$.

(B) Une condition suffisante pour qu'il existe $t \in \mathbb{Z}^{m}$ vérifiant (3.3) est que l'inégalité (3.4) soit satisfaite pour tout $s \in \mathbb{Z}^{n}$ avec $\gamma=1 /(2 \eta)$.

Démonstration. Ce lemme 3.2 n'est autre que le théorème XVII du chapitre $\mathrm{V}, \S 8$ de [3].

Démonstration du lemme 3.1. Pour démontrer l'assertion (i), on utilise la partie (B) du lemme 3.2, avec $C=\eta S^{-1}$ et $X=T$. Soit $\zeta \in \mathbb{R}^{n}$. Comme on a $\left(L_{j}(t)\right)_{1 \leq j \leq n}=t_{1} \gamma_{1}+\ldots+t_{m} \gamma_{m}$ dans $\mathbb{R}^{n}$, il suffit de vérifier, pour tout $s=\left(s_{1}, \ldots, s_{n}\right) \in \mathbb{Z}^{n}$,

$$
\|s \zeta\| \leq \frac{1}{2 \eta} \max \left\{X \max _{1 \leq i \leq m}\left\|M_{i}(s)\right\| ; C \max _{1 \leq j \leq n}\left|s_{j}\right|\right\},
$$

où $s \zeta$ désigne le nombre réel $s_{1} \zeta_{1}+\ldots+s_{n} \zeta_{n}$. Cette inégalité est vraie pour $s=0$. Comme $\|s \zeta\| \leq 1 / 2$, elle est aussi trivialement vérifiée pour les $s \in \mathbb{Z}^{n}$ tels que $\max _{1 \leq j \leq n}\left|s_{j}\right|>S$. Il ne reste plus qu'à considérer les $s \in \mathbb{Z}^{n}$ pour lesquels $0 \neq \max _{1 \leq j \leq n}\left|s_{j}\right| \leq S$, et pour ceux-là on applique l'hypothèse (i) qui donne

$$
\max _{1 \leq i \leq m}\left\|M_{i}(s)\right\| \geq \eta / X
$$

Pour montrer (ii), on raisonne par l'absurde : supposons qu'il existe $\left(s_{1}, \ldots, s_{n}\right) \in \mathbb{Z}^{n}$ vérifiant $0<\max _{1 \leq j \leq n}\left|s_{j}\right| \leq S$ et

$$
\left\|s_{1} \delta_{1}+\ldots+s_{n} \delta_{n}\right\|<\frac{1}{2(n+m) T} .
$$

On choisit $\zeta \in \mathbb{R}^{n}$ tel que $\|s \zeta\|=1 / 2$, et on utilise la partie (A) du lemme 3.2 , avec $C=(2(n+m) S)^{-1}$ et $X=T$ : il n'existe pas de $\left(t_{1}, \ldots, t_{m}\right) \in \mathbb{Z}^{m}$ vérifiant $\max _{1 \leq i \leq m}\left|t_{i}\right| \leq T$ et

$$
\max _{1 \leq j \leq n}\left\|L_{j}(t)-\zeta_{j}\right\| \leq C
$$


R e m ar qu e. Le théorème de Dirichlet ([3], chap. I, th. VI, [16], chap. 2, th. $1 \mathrm{E})$ montre que pour tout $S$ réel $>1$, il existe $\left(s_{1}, \ldots, s_{n}\right) \in \mathbb{Z}^{n}$ vérifiant $0<\max _{1 \leq i \leq n}\left|s_{i}\right| \leq S$, et

$$
\left\|s_{1} \delta_{1}+\ldots+s_{n} \delta_{n}\right\| \leq S^{-n / m} .
$$

Donc l'hypothèse de l'assertion (i) du lemme 3.1 ne peut pas être vérifiée avec un nombre $T$ inférieur à $\eta S^{n / m}$. On vérifie également, grâce au principe des tiroirs, que pour tout entier $T \geq 1$, il existe $\zeta \in \mathbb{R}^{n}$ tel que, pour tout $\left(t_{1}, \ldots, t_{m}\right) \in \mathbb{Z}^{m}$ vérifiant $\max _{1 \leq j \leq m}\left|t_{j}\right| \leq T$, on ait

$$
\left\|\zeta-t_{1} \gamma_{1}-\ldots-t_{m} \gamma_{m}\right\| \geq \frac{1}{2}(2 T+1)^{-m / n} .
$$

Par conséquent, si l'hypothèse de la condition (ii) du lemme 3.1 est vérifiée, alors

$$
S \leq \frac{1}{n+m}(2 T+1)^{m / n} .
$$

Soient $E$ un $\mathbb{R}$-espace vectoriel normé de dimension $n$ et soit $\Omega$ un réseau de $E$. On note $E^{*}=\operatorname{Hom}_{\mathbb{R}}(E, \mathbb{R})$ l'espace vectoriel dual de $E$, et $\Omega^{*}=\{\varphi \in$ $\left.E^{*}: \varphi(\Omega) \subset \mathbb{Z}\right\}$ le réseau dual de $\Omega$ (cf. [2], chap. $7, \S 1, \mathrm{n}^{\circ} 3$ ). Il résulte du théorème de Kronecker qu'un sous-groupe de type fini $Y$ de $E$ contenant $\Omega$ est dense dans $E$ si et seulement si, pour tout $\varphi \in \Omega^{*}$ non nul, on a $\varphi(Y) \not \subset \mathbb{Z}$. Nous allons donner une version quantitative de cet énoncé. On fixe un entier $m \geq 1$ et on pose encore $\eta=2^{-n-m}((n+m) !)^{2}$.

L'énoncé qui suit fait intervenir une fonction réelle de variable réelle $F$, définie et continue sur un intervalle $\left[S_{0}, \infty\right)$ de $\mathbb{R}^{+}$, à valeurs $>1 / \eta$. On suppose $F$ strictement croissante et non bornée $: \lim _{S \rightarrow \infty} F(S)=\infty$. On désigne par $F^{-1}$ la bijection réciproque de $F$, on pose $T_{0}=\eta F\left(S_{0}\right)$ et on définit une fonction $G$, continue et croissante sur l'intervalle $\left[T_{0}, \infty\right)$ et à valeurs réelles positives, par

$$
G(T)=\frac{F^{-1}(T / \eta)}{\eta \sum_{j=1}^{n}\left|\omega_{j}\right|} .
$$

Lemme 3.5. Soient $S_{0}$ un nombre réel positif et $F:\left[S_{0}, \infty\right) \rightarrow \mathbb{R}_{+}$ une fonction continue, strictement croissante, non bornée, à valeurs $>1 / \eta$. Soient $\omega_{1}, \ldots, \omega_{n}$ des éléments de $\Omega$ linéairement indépendants (sur $\mathbb{Z}$ ou sur $\mathbb{R}$, c'est équivalent), et soient $y_{1}, \ldots, y_{m}$ des éléments de $E$. On suppose que pour tout $\varphi \in \Omega^{*}$ non nul, si on pose

$$
S=\max \left\{\left|\varphi\left(\omega_{1}\right)\right|, \ldots,\left|\varphi\left(\omega_{n}\right)\right| ; S_{0}\right\},
$$

on a

$$
\max _{1 \leq i \leq m}\left\|\varphi\left(y_{i}\right)\right\| \geq 1 / F(S) .
$$


Alors pour tout $x \in E$ et pour nombre réel $T \geq T_{0}$, il existe $\omega \in \Omega$ et $\left(t_{1}, \ldots, t_{m}\right) \in \mathbb{Z}^{m}$ vérifiant

$$
\max \left\{\left|t_{1}\right|, \ldots,\left|t_{m}\right|\right\} \leq T \quad \text { et } \quad\left|x-\omega-t_{1} y_{1}-\ldots-t_{m} y_{m}\right| \leq 1 / G(T) .
$$

En posant $\varepsilon_{0}=S_{0}^{-1} \eta \sum_{j=1}^{n}\left|\omega_{j}\right|$, on peut écrire la conclusion sous la forme suivante : pour tout $x \in E$ et pour tout $\varepsilon$ dans l'intervalle $0<\varepsilon<\varepsilon_{0}$, il existe $\omega \in \Omega$ et $\left(t_{1}, \ldots, t_{m}\right) \in \mathbb{Z}^{m}$ vérifiant

$$
\left|x-\omega-t_{1} y_{1}-\ldots-t_{m} y_{m}\right| \leq \varepsilon
$$

avec

$$
\max \left\{\left|t_{1}\right|, \ldots,\left|t_{m}\right|\right\} \leq \eta F(S), \quad \text { ò̀ } \quad S=\varepsilon^{-1} \eta \sum_{j=1}^{n}\left|\omega_{j}\right| .
$$

Démonstration. Pour démontrer le lemme 3.5 , on écrit $y_{1}, \ldots, y_{m}$ dans la base $\omega_{1}, \ldots, \omega_{n}$ de $E$ :

$$
y_{i}=\sum_{j=1}^{n} \vartheta_{j i} \omega_{j} \quad(1 \leq i \leq m) .
$$

On va utiliser la partie (i) du lemme 3.1. Soit $S$ un nombre réel $\geq S_{0}$ et soit $s \in \mathbb{Z}^{n}$ vérifiant $0<\max _{1 \leq j \leq n}\left|s_{j}\right| \leq S$. On définit $\varphi \in \Omega^{*} \operatorname{par} \varphi\left(\omega_{j}\right)=s_{j}$ $(1 \leq j \leq n)$. Alors

$$
\varphi\left(y_{i}\right)=\sum_{j=1}^{n} \vartheta_{j i} s_{j} \quad(1 \leq i \leq m) .
$$

On a par hypothèse

$$
\max _{1 \leq i \leq m}\left\|\sum_{j=1}^{n} \vartheta_{j i} s_{j}\right\| \geq 1 / F(S),
$$

ce qui permet d'appliquer le lemme 3.1. Soit $x \in E$, soit $T$ un nombre réel $\geq T_{0}$, et soit $S$ le nombre réel défini par $F(S)=T / \eta$. On écrit $x=$ $\zeta_{1} \omega_{1}+\ldots+\zeta_{n} \omega_{n}$ avec $\left(\zeta_{1}, \ldots, \zeta_{n}\right) \in \mathbb{R}^{n}$. Alors il existe $t \in \mathbb{Z}^{m}$ vérifiant $\max _{1 \leq i \leq m}\left|t_{i}\right| \leq T$ et

$$
\max _{1 \leq j \leq n}\left\|\zeta_{j}-\sum_{i=1}^{m} \vartheta_{j i} t_{i}\right\| \leq \eta / S
$$

Autrement dit, il existe $a=\left(a_{1}, \ldots, a_{n}\right) \in \mathbb{Z}^{n}$ tel que

$$
\max _{1 \leq j \leq n}\left|\zeta_{j}-a_{j}-\sum_{i=1}^{m} t_{i} \vartheta_{j i}\right| \leq \eta / S .
$$

On pose alors $\omega=a_{1} \omega_{1}+\ldots+a_{n} \omega_{n}$ et on utilise la relation

$$
\frac{\eta}{S} \sum_{j=1}^{n}\left|\omega_{j}\right|=\frac{1}{G(T)}
$$


Re m ar ques. 1. Si l'hypothèse du lemme 3.5 est vraie pour une fonction $F$, alors elle est encore vraie pour toute fonction qui majore $F$. Ainsi il est quelquefois plus simple d'énoncer la conclusion non pas pour la fonction $G$ elle même, mais pour une fonction minorant $G$.

2. Plusieurs types de fonctions $F$ interviendront.

- Le cas le plus favorable est celui où l'hypothèse est vraie avec $F(S)=c S^{\kappa}$ pour $S \geq S_{0}$ (où $S_{0}, c$ et $\kappa$ sont trois constantes); l'exposant $\kappa$ est alors nécessairement $\geq n / m$. Dans ce cas on a $G(T)=c^{\prime} T^{\theta}$ pour $T \geq T_{0}$ avec $\theta=1 / \kappa$ et deux autres constantes $T_{0}$ et $c^{\prime}$. En particulier on a $\theta \leq m / n$. Dans ces circonstances, le nombre $\theta+1$ est le coefficient de densité de [19], chap. I, §3; il est majoré par $l / n$, où $l$ est le rang de $\Omega+Y$ sur $\mathbb{Z}$. Le lemme 1.3.7 de [19] donne une majoration de ce coefficient introduisant des conditions algébriques (comparer à l'hypothèse du corollaire 2.4), et non diophantiennes, sur la répartition de $\Omega+Y$ dans $\mathbb{R}^{n}$.

- Si l'hypothèse du lemme 2.3 est satisfaite pour une fonction $F(S)=$ $\exp \left\{c(\log S)^{\kappa}\right\}$ avec $\kappa \geq 1$, alors la conclusion est vraie pour une fonction $G$ de la forme $G(T)=\exp \left\{c^{\prime}(\log T)^{\theta}\right\}$ pour $T \geq T_{0}$, avec $\theta=1 / \kappa$.

- Enfin, dans les cas moins favorables, on aura seulement $F(S)=$ $\exp \left(c S^{\kappa}\right)$, avec $\kappa>0$, donc $G(T)=c^{\prime}(\log T)^{\theta}$ avec $\theta=1 / \kappa$.

(b) Variante réelle du lemme de transfert. Le lemme 3.5 est bien adapté au cas où l'espace réel ambiant contient un réseau apparaissant de façon naturelle. Quand il n'y a pas de réseau naturel, on peut appliquer la variante suivante, dans laquelle $|\cdot|$ désigne la norme $|x|=\max _{1 \leq i \leq n}\left|x_{i}\right| \operatorname{sur} \mathbb{R}^{n}$.

Lemme 3.6. Soient $y_{1}, \ldots, y_{l}$ des éléments de $\mathbb{R}^{n}, S_{0}$ un nombre réel positif et $F:\left[S_{0}, \infty\right) \rightarrow \mathbb{R}_{+}$une fonction réelle de variable réelle, continue, croissante et non bornée. On suppose que pour toute forme linéaire $\phi \in$ $\operatorname{Hom}_{\mathbb{R}}\left(\mathbb{R}^{n}, \mathbb{R}\right)$ satisfaisant $\mathrm{N}(\phi) \geq S_{0}$, on a

$$
\max _{1 \leq j \leq l}\left\|\phi\left(y_{j}\right)\right\| \geq 1 / F(S) \quad \text { avec } S=\mathrm{N}(\phi) .
$$

Sous ces hypothèses il existe des constantes $T_{0}, C_{1}$ et $C_{2}$ positives telles que, si on pose $G(T)=C_{1} F^{-1}\left(C_{2} T\right)$ pour $T \geq T_{0}$, alors pour tout $x \in \mathbb{R}^{n}$ et tout $T \geq T_{0}(1+|x|)$, le système d'inéquations

$$
\max _{1 \leq j \leq l}\left|t_{j}\right| \leq T \quad \text { et } \quad\left|x-t_{1} y_{1}-\ldots-t_{l} y_{l}\right| \leq 1 / G(T)
$$

admet une solution $t \in \mathbb{Z}^{l}$.

Démonstration. Le sous-groupe $Y=\mathbb{Z} y_{1}+\ldots+\mathbb{Z} y_{l}$ est dense dans $\mathbb{R}^{n}$ : en effet, l'hypothèse implique que pour tout $\varphi \in \operatorname{Hom}_{\mathbb{R}}\left(\mathbb{R}^{n}, \mathbb{R}\right), \varphi \neq 0$, on a $\varphi(Y) \not \subset \mathbb{Z}$. En particulier, $\left\{y_{1}, \ldots, y_{l}\right\}$ contient une base de $\mathbb{R}^{n}$. Il n'y 
a donc pas de restriction à supposer que $y_{l-n+1}, \ldots, y_{l}$ sont linéairement indépendants sur $\mathbb{R}$. On pose $m=l-n, \omega_{i}=y_{m+i}(1 \leq i \leq n)$ et $\Omega=$ $\mathbb{Z} \omega_{1}+\ldots+\mathbb{Z} \omega_{n}$. Comme $\Omega^{*}$ est un réseau de $\operatorname{Hom}_{\mathbb{R}}\left(\mathbb{R}^{n}, \mathbb{R}\right)$, il existe un nombre réel $c_{7}>0$ tel que, pour tout élément non nul $\varphi$ de $\Omega^{*}$, on ait $\mathrm{N}(\varphi) \geq c_{7}$. Soit $N_{0}$ un entier positif vérifiant $N_{0} \geq S_{0} / c_{7}$.

On va utiliser le lemme 3.5. Pour en vérifier l'hypothèse, on considère un élément non nul $\varphi$ de $\Omega^{*}$, et on pose $\phi=N_{0} \varphi$. Alors le nombre $S:=\mathrm{N}(\phi)=$ $N_{0} \mathrm{~N}(\varphi)$ satisfait $S \geq S_{0}$. On a aussi $S \leq c_{8} \max _{1 \leq i \leq n}\left|\varphi\left(\omega_{i}\right)\right|$, avec une constante $c_{8}$ qui ne dépend que de $y_{1}, \ldots, y_{l}$. On pose encore $\widetilde{S}_{0}=c_{8}^{-1} S_{0}$, $\widetilde{S}=\max \left\{\left|\varphi\left(\omega_{1}\right)\right|, \ldots,\left|\varphi\left(\omega_{n}\right)\right|, \widetilde{S}_{0}\right\}$ et on définit une fonction $\widetilde{F}:\left[\widetilde{S}_{0}, \infty\right) \rightarrow$ $\mathbb{R}^{+}$par $\widetilde{F}(\widetilde{S})=N_{0} F\left(c_{8} \widetilde{S}\right)$. Alors

$$
\max _{1 \leq j \leq l}\left\|\varphi\left(y_{j}\right)\right\|=N_{0}^{-1} \max _{1 \leq j \leq l}\left\|\phi\left(y_{j}\right)\right\| \geq \frac{1}{N_{0} F(S)} \geq \frac{1}{N_{0} F\left(c_{8} \widetilde{S}\right)}=\frac{1}{\widetilde{F}(\widetilde{S})} .
$$

Les hypothèses du lemme 3.5 sont donc vérifiées pour la fonction $\widetilde{F}$. Par conséquent, il existe une constante $c_{9} \geq 1$ telle que, pour tout $T_{1} \geq c_{9}$ et tout $x \in \mathbb{R}^{n}$, il existe $t \in \mathbb{Z}^{l}$ avec

$$
\max _{1 \leq j \leq m}\left|t_{j}\right| \leq T_{1} \quad \text { et } \quad\left|x-t_{1} y_{1}-\ldots-t_{l} y_{l}\right| \leq 1 / \widetilde{G}\left(T_{1}\right),
$$

avec une fonction $\widetilde{G}$ de la forme

$$
\widetilde{G}\left(T_{1}\right)=c_{10} \widetilde{F}^{-1}\left(c_{11} T_{1}\right) .
$$

On majore $\max _{m+1 \leq j \leq l}\left|t_{j}\right|$ par $c_{12}\left(T_{1}+|x|\right)$ et on pose $T_{0}=2 c_{9} c_{12}$. Pour $T \geq T_{0}(1+|x|)$, on peut appliquer ce qui vient d'être démontré avec $T_{1}=T / 2 c_{12}$. On obtient ainsi le résultat annoncé avec $C_{1}=c_{10}$ et $C_{2}=$ $c_{11} /\left(2 c_{12}\right)$.

(c) Variante complexe du lemme de transfert. Soient $r_{1}$ et $r_{2}$ des entiers $\geq 0$ avec $\left(r_{1}, r_{2}\right) \neq(0,0)$. On pose $n=r_{1}+r_{2}$ et $d=r_{1}+2 r_{2}$. Pour $\xi \in \mathbb{R}^{r_{1}} \times \mathbb{C}^{r_{2}}$ on pose $|\xi|=\max _{1 \leq i \leq n}\left|\xi_{i}\right|$. On désigne enfin par $\bar{z}$ le complexe conjugué de $z \in \mathbb{C}$.

L'énoncé suivant généralise le lemme 3.6 (qui correspond au cas $r_{2}=0$, $n=d=r_{1}$ ); c'est un analogue quantitatif de la variante complexe du théorème de Kronecker de [22], th. 5.1 (voir aussi [23], chap. II, §6).

Pour $\sigma=\left(\sigma_{1}, \ldots, \sigma_{n}\right) \in \mathbb{R}^{r_{1}} \times \mathbb{C}^{r_{2}}$, on définit $\psi_{\sigma}: \mathbb{R}^{r_{1}} \times \mathbb{C}^{r_{2}} \rightarrow \mathbb{R}$ par

$$
\psi_{\sigma}(\xi)=\sigma_{1} \xi_{1}+\ldots+\sigma_{n} \xi_{n}+\bar{\sigma}_{r_{1}+1} \bar{\xi}_{r_{1}+1}+\ldots+\bar{\sigma}_{n} \bar{\xi}_{n} .
$$

Proposition 3.7. Soient $y_{1}, \ldots, y_{l}$ des éléments de $\mathbb{R}^{r_{1}} \times \mathbb{C}^{r_{2}}$ et $F$ : $\left[S_{0}, \infty\right) \rightarrow \mathbb{R}_{+}$une fonction réelle de variable réelle, continue, croissante et non bornée. On suppose que, pour tout $\sigma=\left(\sigma_{1}, \ldots, \sigma_{n}\right) \in \mathbb{R}^{r_{1}} \times \mathbb{C}^{r_{2}}$ vérifiant

$$
S:=\max \left\{\left|\sigma_{1}\right|, \ldots,\left|\sigma_{n}\right|\right\} \geq S_{0},
$$


on $a$

$$
\max _{1 \leq j \leq l}\left\|\psi_{\sigma}\left(y_{j}\right)\right\| \geq 1 / F(S)
$$

Il existe des constantes $T_{0}, C_{1}$ et $C_{2}$ positives possédant la propriété suivante : si on pose $G(T)=C_{1} F^{-1}\left(C_{2} T\right)$ pour $T \geq T_{0}$, alors pour tout $\xi \in$ $\mathbb{R}^{r_{1}} \times \mathbb{C}^{r_{2}}$ et tout $T \geq T_{0}(1+|\xi|)$, le système d'inéquations

$$
\max _{1 \leq j \leq l}\left|t_{j}\right| \leq T \quad \text { et } \quad\left|\xi-t_{1} y_{1}-\ldots-t_{l} y_{l}\right| \leq 1 / G(T)
$$

admet une solution $t \in \mathbb{Z}^{l}$.

Démonstration. On désigne par $E$ l'espace vectoriel réel normé $\mathbb{R}^{r_{1}} \times \mathbb{C}^{r_{2}}$. On définit une application $\mathbb{R}$-linéaire $\theta: E \rightarrow \mathbb{R}^{r_{1}} \times \mathbb{C}^{2 r_{2}}$ par $\theta(x, z)=(x, z, \bar{z})$ et un isomorphisme $\mathbb{R}$-linéaire $\chi: E \rightarrow \mathbb{R}^{d}$ par $\chi(x, z)=(x, \Re(z), \Im(z))$. On définit encore $\widetilde{y}_{j}=\chi\left(y_{j}\right)(1 \leq j \leq l)$. On va vérifier l'hypothèse du lemme 3.6 (avec $n$ remplacé par $d$ ) pour le sousgroupe de $\mathbb{R}^{d}$ engendré par $\widetilde{y}_{1}, \ldots, \widetilde{y}_{l}$. Pour cela, soit $\phi$ une forme linéaire non nulle dans $\operatorname{Hom}_{\mathbb{R}}\left(\mathbb{R}^{d}, \mathbb{R}\right)$. On désigne par $\left(e_{1}, \ldots, e_{d}\right)$ la base canonique de $\mathbb{R}^{d}$, on pose $\widetilde{\sigma}_{i}=\phi\left(e_{i}\right)(1 \leq i \leq d)$ et on définit $\sigma=\left(\sigma_{1}, \ldots, \sigma_{n}\right) \in E$ par

$$
\sigma_{\nu}= \begin{cases}\widetilde{\sigma}_{\nu} & \text { pour } 1 \leq \nu \leq r_{1}, \\ \frac{1}{2}\left(\widetilde{\sigma}_{\nu}-i \widetilde{\sigma}_{r_{2}+\nu}\right) & \text { pour } r_{1}<\nu \leq n,\end{cases}
$$

de sorte que $\psi_{\sigma}=\phi \circ \chi$.

L'hypothèse de la proposition 3.7 concernant $\max _{1 \leq j \leq l}\left\|\psi_{\sigma}\left(\widetilde{y}_{j}\right)\right\|$ permet donc de vérifier l'hypothèse correspondante du lemme 3.6 portant sur $\max _{1 \leq j \leq l}\left\|\phi\left(y_{j}\right)\right\|$. On déduit du lemme 3.6 l'existence de constantes $\widetilde{C}_{1}$, $C_{2}$ et $T_{0}$ telles que, si on pose $\widetilde{G}(T)=\widetilde{C}_{1} F^{-1}\left(C_{2} T\right)$ pour $T \geq T_{0}$, alors pour tout $\xi \in E$ et pour tout $T \geq T_{0}(1+|x|)$ avec $x=\chi(\xi)$, le système d'inéquations

$$
\max _{1 \leq j \leq l}\left|t_{j}\right| \leq T \quad \text { et } \quad\left|x-t_{1} \widetilde{y}_{1}-\ldots-t_{l} \widetilde{y}_{l}\right| \leq 1 / \widetilde{G}(T)
$$

admet une solution $t \in \mathbb{Z}^{l}$. On prend $C_{1}=\widetilde{C}_{1} / \sqrt{2}$, de sorte que $G(T)=$ $\widetilde{G}(T) / \sqrt{2}$, et on peut conclure, pour $T \geq T_{0}(1+|\xi|)$,

$$
\left|\xi-t_{1} y_{1}-\ldots-t_{l} y_{l}\right| \leq \sqrt{2} / \widetilde{G}(T)=1 / G(T)
$$

4. Approximation diophantienne sur les tores. Ce paragraphe est consacré à la démonstration du théorème 1.2 et à celle du corollaire 1.3.

Commençons par une remarque concernant l'hypothèse $d \geq 2$ du théorème 1.2. Pour $d=1$ on peut écrire $\varphi(z)=\lambda z$ avec un nombre complexe non nul $\lambda$ de module $\mathrm{N}(\varphi)$. Comme $l \geq d^{2}-d+1=1$ et que $y_{1}, \ldots, y_{l}$ sont linéairement indépendants, on a $y_{j} \neq 0$ pour $1 \leq j \leq l$. Posons $\alpha_{j}=e^{y_{j}}$. L'inégalité de Liouville entraîne, pour $1 \leq j \leq l$, soit $\alpha_{j}=1$, soit $\left|\alpha_{j}-1\right| \geq$ 
$2\left(2 e^{\mathrm{h}\left(\alpha_{j}\right)}\right)^{-D}$. Comme $y_{j} \neq 0$, on en déduit $\left|y_{j}\right| \geq\left(2 e^{\mathrm{h}\left(\alpha_{j}\right)}\right)^{-D}$, ce qui donne

$$
\left|\varphi\left(y_{j}\right)\right| \geq \mathrm{N}(\varphi) 2^{-D} A^{-D} \quad(1 \leq j \leq l) .
$$

Cette inégalité est légèrement moins précise que ce que donnerait le théorème 1.2 avec $\kappa_{0}=1$ et $c_{0}=l$. C'est pourquoi nous avons supposé $d \geq 2$. Si, pour $d=1$, on impose $A \geq 2$, alors la conclusion du théorème 1.2 est encore vraie avec $\kappa_{0}=\kappa_{0}(1, l)=1$ et $c_{0}=c_{0}(1, l)=2$.

Voici comment interviendra la condition (IL). Soit $d$ un entier positif. On désigne par $G$ le groupe algébrique $\mathbb{G}_{\mathrm{m}}^{d}$ et par $\exp _{G}$ son application exponentielle. En identifiant l'espace tangent à l'origine de $G(\mathbb{C})$ à $\mathbb{C}^{d}$, on écrit

$$
\exp _{G}: \mathbb{C}^{d} \rightarrow\left(\mathbb{C}^{\times}\right)^{d}, \quad\left(z_{1}, \ldots, z_{d}\right) \mapsto\left(e^{z_{1}}, \ldots, e^{z_{d}}\right) .
$$

Lemme 4.2. Soit $Y$ un sous-groupe de type fini de $\mathbb{C}^{d}$ possédant la propriété (IL). Si $G^{*}$ est un sous-groupe algébrique connexe de $G$, distinct de $G$, tel que $Y \cap \exp _{G}^{-1}\left(G^{*}(\mathbb{C})\right) \neq\{0\}$, alors la codimension de $G^{*}$ dans $G$ est 1 , et

$$
\operatorname{rang}_{\mathbb{Z}}\left(Y \cap \exp _{G}^{-1}\left(G^{*}(\mathbb{C})\right)\right) \leq 1 .
$$

Remarque. Notons $\Gamma=\exp _{G} Y \subset\left(\mathbb{C}^{\times}\right)^{d}$. L'image par $\exp _{G}$ de $Y \cap$ $\exp _{G}^{-1}\left(G^{*}(\mathbb{C})\right)$ est $\Gamma \cap G^{*}(\mathbb{C})$, donc

$$
\operatorname{rang}_{\mathbb{Z}}\left(\Gamma \cap G^{*}(\mathbb{C})\right) \leq \operatorname{rang}_{\mathbb{Z}}\left(Y \cap \exp _{G}^{-1}\left(G^{*}(\mathbb{C})\right)\right) .
$$

En particulier, la condition $\Gamma \cap G^{*}(\mathbb{C}) \neq\{1\}$ entraîne $Y \cap \exp _{G}^{-1}\left(G^{*}(\mathbb{C})\right)$ $\neq\{0\}$.

Démonstration du lemme 4.2. Les hypothèses du lemme 4.2 impliquent clairement $d \geq 2$. On désigne par $\Omega=(2 i \pi \mathbb{Z})^{d}$ le noyau de $\exp _{G}$. Comme $Y$ possède la propriété (IL), on a $Y \cap \Omega=\{0\}$. L'espace tangent à l'origine de $G^{*}$ est un sous-espace de celui de $G$; on a identifié les points complexes du second à $\mathbb{C}^{d}$; ceux du premier forment alors un sous-espace $W$ de $\mathbb{C}^{d}$, rationnel sur $\mathbb{Q}$, de dimension $\operatorname{dim}_{\mathbb{C}} W=\operatorname{dim} G^{*}$. De plus, $G^{*}$ étant connexe, on a $\exp _{G}^{-1}\left(G^{*}(\mathbb{C})\right)=W+\Omega$.

Soit $y \in Y \cap(W+\Omega)$ : il existe $\omega \in \Omega$ tel que $y+\omega \in W$. Alors $y$ appartient à $W+\mathbb{C} \omega$, qui est un sous-espace vectoriel de $\mathbb{C}^{d}$ rationnel sur $\mathbb{Q}$. Si $G^{*}$ est de codimension $>1$ dans $G$, alors $W+\mathbb{C} \omega \neq \mathbb{C}^{d}$, donc $y=0$. Si $G^{*}$ est de codimension 1 et si $y_{1}$ et $y_{2}$ sont deux éléments de $Y \cap(W+\Omega)$, on écrit, pour $j=1$ et $j=2$,

$$
y_{j}+\omega_{j} \in W \quad \text { avec } \omega_{j} \in \Omega
$$

Etant donné que $W$ est rationnel sur $\mathbb{Q}$, le $\mathbb{Z}$-module $\Omega / \Omega \cap W$ est de rang 1. Par conséquent, il existe $s=\left(s_{1}, s_{2}\right) \in \mathbb{Z}^{2}, s \neq(0,0)$, tel que $s_{1} \omega_{1}+s_{2} \omega_{2} \in \Omega \cap W$. Alors $s_{1} y_{1}+s_{2} y_{2} \in Y \cap W=\{0\}$, et finalement $s_{1} y_{1}+s_{2} y_{2}=0$. 
Nous déduirons le théorème 1.2 de l'énoncé suivant, qui sera utilisé aussi dans la démonstration du théorème 1.4. On reprend les notations communes aux théorèmes 1.2 et 1.4 , avec les entiers $d$ et $l$, le sous-groupe $Y$ de $\mathcal{L}^{d}$, le corps de nombres $K$ de degré $D$, le paramètre $A$ et la forme linéaire $\varphi$.

ThÉORÈme 4.3. Soit $S$ un entier positif. On définit

$$
V=4((12 d+13) d l S D \log A)^{d} .
$$

Alors le nombre d'éléments de l'ensemble

$$
\begin{aligned}
\mathcal{E}=\left\{\left(s_{1}, \ldots, s_{l}\right)\right. & \in \mathbb{Z}^{l}: \\
\left|s_{j}\right| & \left.<S(1 \leq j \leq l) ;\left|\varphi\left(s_{1} y_{1}+\ldots+s_{l} y_{l}\right)\right| \leq \mathrm{N}(\varphi) e^{-V}\right\}
\end{aligned}
$$

est majoré par Card $\mathcal{E} \leq 4 V^{d-1}$.

Démonstration. Quitte à diviser $\varphi$ par $\mathrm{N}(\varphi)$, il n'y a pas de restriction à supposer $\mathrm{N}(\varphi)=1$, c'est-à-dire

$$
\varphi(z)=\vartheta_{1} z_{1}+\ldots+\vartheta_{d} z_{d} \quad \text { avec } \max \left\{\left|\vartheta_{1}\right|, \ldots,\left|\vartheta_{d}\right|\right\}=1 .
$$

Quitte ensuite à permuter éventuellement les variables et à diviser par $-\vartheta_{d}$, on peut encore supposer $\vartheta_{d}=-1$ :

$$
\varphi(z)=\vartheta_{1} z_{1}+\ldots+\vartheta_{d-1} z_{d-1}-z_{d} .
$$

On va utiliser le théorème 2.1 de [24] avec

$$
\begin{gathered}
l_{0}=d_{0}=0, \quad l_{1}=l, \quad d_{1}=d, \quad r=r_{3}=d-1, \quad r_{1}=r_{2}=0, \\
W=\mathcal{W}^{\prime}=\{0\}, \quad \log A_{i}=l S \log A, \quad E=e \quad \text { et } \quad M=\text { Card } \mathcal{E} .
\end{gathered}
$$

On pose $U=V /(12 d+13)$ et on définit des nombres réels $A_{1}, \ldots, A_{d}$ et des entiers positifs $T_{1}, \ldots, T_{d}$ par

$\log A_{i}=l S \log A, \quad T_{i}=\left[U /\left(d D \log A_{i}\right)\right]=[U /(d l S D \log A)] \quad(1 \leq i \leq d)$.

On vérifie, grâce aux choix de $U$ et $V$,

$$
(U /(d l S D \log A))^{d}=4 V^{d-1},
$$

donc

$$
\left(T_{1}+1\right) \ldots\left(T_{d}+1\right) \geq 4 V^{d-1},
$$

ce qui est l'hypothèse "principale" du $\S 2 \mathrm{~g}$ de [24]. On a aussi $T_{1} \ldots T_{d} \leq$ $4 V^{d-1}$.

On définit $y_{1}^{\prime}, \ldots, y_{l}^{\prime}$ dans $\mathbb{C}^{d}$ par $y_{j}^{\prime}=\left(u_{1 j}^{\prime}, \ldots, u_{d j}^{\prime}\right)(1 \leq j \leq l)$, avec

$$
u_{i j}^{\prime}= \begin{cases}u_{i j} & \text { pour } 1 \leq i \leq d-1, \\ \vartheta_{1} u_{1 j}+\ldots+\vartheta_{d-1} u_{d-1, j} & \text { pour } i=d,\end{cases}
$$

de sorte que

$$
y_{j}^{\prime}-y_{j}=\left(0, \ldots, 0, \varphi\left(y_{j}\right)\right) \quad(1 \leq j \leq l) .
$$


Pour chaque $s=\left(s_{1}, \ldots, s_{l}\right) \in \mathcal{E}$, on définit deux éléments $\eta_{s}$ et $\eta_{s}^{\prime}$ de $\mathbb{C}^{d}$ :

$$
\eta_{s}=s_{1} y_{1}+\ldots+s_{l} y_{l} \quad \text { et } \quad \eta_{s}^{\prime}=s_{1} y_{1}^{\prime}+\ldots+s_{l} y_{l}^{\prime} .
$$

Les points $\eta_{s}^{\prime}(s \in \mathcal{E})$ appartiennent à l'hyperplan $\mathcal{V}^{\prime}=\operatorname{Ker} \varphi$ de $\mathbb{C}^{d}$ et on a

$$
\max _{s \in \mathcal{E}}\left|\eta_{s}-\eta_{s}^{\prime}\right| \leq e^{-V}
$$

Le sous-groupe $\Gamma$ de $\left(\mathbb{C}^{\times}\right)^{d}$ engendré par l'ensemble

$$
\Sigma=\left\{\left(\alpha_{i 1}^{s_{1}} \ldots \alpha_{i l}^{s_{l}}\right)_{1 \leq i \leq d}: s \in \mathcal{E}\right\}
$$

est $\exp _{G} Y$.

On applique maintenant le théorème 2.1 de [24] au groupe algébrique $G=\mathbb{G}_{\mathrm{m}}^{d}$ : il existe un sous-groupe algébrique connexe $G^{*}$, de codimension $d^{*} \geq 1$ dans $G$, tel que l'image

$$
\Sigma^{*}=\left(\Sigma+G^{*}(K)\right) / G^{*}(K)
$$

de $\Sigma$ par la surjection canonique $G(K) \rightarrow G(K) / G^{*}(K)$ satisfasse

$$
\operatorname{Card} \Sigma^{*} \leq T_{1}^{d^{*}} \text {. }
$$

Si Card $\Sigma^{*}=$ Card $\mathcal{E}$, comme $d^{*} \leq d$ et que $T_{1}^{d} \leq 4 V^{d-1}$, l'estimation annoncée est bien vraie.

Sinon, on a $Y \cap \exp _{G}^{-1}\left(G^{*}(K)\right) \neq\{0\}$. Alors $d \geq 2$; de plus le lemme 4.2 montre que la codimension $d^{*}$ de $G^{*}$ dans $G$ est 1 , et que le rang sur $\mathbb{Z}$ de $Y \cap \exp _{G}^{-1}\left(G^{*}(K)\right)$ est $\leq 1$. En utilisant encore la propriété (IL), on voit que la restriction à $Y$ de l'application $\exp _{G}$ est injective, donc Card $\Sigma^{*} \geq$ $($ Card $\mathcal{E}) /(2 S)$. Enfin la minoration $U>2 d l S^{2} D \log A$ entraîne $T_{1} \geq 2 S$. On obtient ainsi

$$
\text { Card } \mathcal{E} \leq 2 S \text { Card } \Sigma^{*} \leq 2 T_{1} S \leq T_{1}^{2} \leq T_{1}^{d},
$$

ce qui termine la démonstration du théorème 4.3 .

Démonstration du théorème 1.2. On définit $S$ comme le plus petit entier positif tel que

$$
S^{l-d^{2}+d}>4^{d}((12 d+13) d l D \log A)^{d(d-1)} .
$$

Par conséquent, le paramètre $V$ du théorème 4.3 satisfait $4 V^{d-1}<(2 S-1)^{l}$. On en déduit qu'il existe $\left(s_{1}, \ldots, s_{l}\right) \in \mathbb{Z}^{l}$, avec $\left|s_{j}\right|<S(1 \leq j \leq l)$, tel que

$$
\left|\varphi\left(s_{1} y_{1}+\ldots+s_{l} y_{l}\right)\right| \geq \mathrm{N}(\varphi) e^{-V} .
$$

On en déduit

$$
\max _{1 \leq j \leq l}\left|\varphi\left(y_{j}\right)\right| \geq \frac{1}{l S} \mathrm{~N}(\varphi) e^{-V} .
$$

De la définition de $S$ résulte $S>1$, donc $S-1 \geq S / 2$ et

$$
(S / 2)^{l-d^{2}+d} \leq 4^{d}((12 d+13) d l D \log A)^{d(d-1)},
$$


ce qui donne

$$
\left(V / 2^{d}\right)^{l-d^{2}+d} \leq 4^{d+l}((12 d+13) d l D \log A)^{d l} .
$$

Nous allons montrer que cette majoration implique

$$
V+\log (l S) \leq c_{0}(D \log A)^{\kappa_{0}}
$$

ce qui donnera la minoration désirée :

$$
\max _{1 \leq j \leq l}\left|\varphi\left(y_{j}\right)\right| \geq \mathrm{N}(\varphi) \exp \left\{-c_{0}(D \log A)^{\kappa_{0}}\right\} .
$$

On majore $\log (l S)$ assez brutalement en utilisant (4.4) avec les conditions $d \geq 2, l \geq d^{2}-d+1, D \log A \geq e$, ainsi que l'inégalité $\kappa_{0} \geq 1$ :

$$
\log (l S) \leq d^{3} l(D \log A)^{\kappa_{0}} .
$$

Pour établir (4.5), il ne reste plus qu'à vérifier que la constante $c_{0}=d^{7 d^{3}} l^{d}$ du théorème 1.2 satisfait

$$
\left(\frac{c_{0}-d^{3} l}{2^{d}}\right)^{l-d(d-1)}>4^{d+l}((12 d+13) d l)^{d l},
$$

ce qui ne demande qu'un peu de calcul. C'est d'ailleurs la seule contrainte que doive satisfaire $c_{0}$; on peut donc raffiner légèrement l'estimation numérique du théorème 1.2, mais il est apparu préférable de donner un énoncé simple.

Démonstration du corollaire 1.3. Pour $1 \leq j \leq l$, soit $s_{j}$ un élément de $\mathbb{Z}$ à distance minimale de $\varphi\left(y_{j}\right)$ :

$$
\left\|\varphi\left(y_{j}\right)\right\|=\left|\varphi\left(y_{j}\right)-s_{j}\right| \quad(1 \leq j \leq l) .
$$

Commençons par le cas $s_{1}=\ldots=s_{l}=0$. Alors $\left\|\varphi\left(y_{j}\right)\right\|=\left|\varphi\left(y_{j}\right)\right|(1 \leq j \leq$ l). Si, de plus, $d \geq 2$, alors on peut utiliser directement le théorème 1.2 :

$$
\max _{1 \leq j \leq l}\left|\varphi\left(y_{j}\right)\right| \geq \mathrm{N}(\varphi) \exp \left\{-c_{0}(D \log A)^{\kappa_{0}}\right\}
$$

avec $c_{0}=c_{0}(d, l)$ et $\kappa_{0}=\kappa_{0}(d, l)$. Comme $N \geq 1, \kappa_{1} \geq \kappa_{0}>0$ et $c_{1} \geq$ $c_{0}(D \log A)^{\kappa_{0}}$, on peut conclure

$$
\max _{1 \leq j \leq l}\left\|\varphi\left(y_{j}\right)\right\| \geq \mathrm{N}(\varphi) e^{-c_{1}} \geq \mathrm{N}(\varphi) \exp \left\{-c_{1} N^{\kappa_{1}}\right\} .
$$

Toujours dans le cas $s_{1}=\ldots=s_{l}=0$, si $d=1$, on applique l'inégalité de Liouville (4.1) et on conclut grâce aux minorations

$$
c_{1} N^{\kappa_{1}} \geq c_{1} \geq D \log 2+D \log A .
$$

On suppose maintenant $s_{l} \neq 0$. On va utiliser le théorème 1.2 avec $l$ remplacé par $\widetilde{l}=l-1$ et avec les $y_{j}$ remplacés par $\widetilde{y}_{j}=s_{l} y_{j}-s_{j} y_{l}(1 \leq j \leq \widetilde{l})$.

Le sous-groupe $\widetilde{Y}=\mathbb{Z} \widetilde{y}_{1}+\ldots+\mathbb{Z} \widetilde{y}_{\tilde{l}}$ de $\mathbb{C}^{d}$ possède encore la propriété (IL). On a

$$
\varphi\left(\widetilde{y}_{j}\right)=s_{l}\left(\varphi\left(y_{j}\right)-s_{j}\right)-s_{j}\left(\varphi\left(y_{l}\right)-s_{l}\right),
$$


donc, si on pose $S=\max \left\{\left|s_{1}\right|, \ldots,\left|s_{l}\right|\right\}$, on a

$$
\max _{1 \leq j \leq \tilde{l}}\left|\varphi\left(\widetilde{y}_{j}\right)\right| \leq 2 S \max _{1 \leq k \leq l}\left\|\varphi\left(y_{k}\right)\right\| .
$$

Si on écrit, pour $1 \leq j \leq \widetilde{l}$,

$$
\widetilde{y}_{j}=\left(\widetilde{u}_{i j}\right)_{1 \leq i \leq d} \quad \text { et } \quad \widetilde{\alpha}_{i j}=e^{\tilde{u}_{i j}} \quad(1 \leq i \leq d),
$$

on a

$$
\max \left\{e / D, \mathrm{~h}\left(\widetilde{\alpha}_{i j}\right),(e / D)\left|\widetilde{u}_{i j}\right|\right\} \leq 2 S \log A,
$$

ce qui nous autorise à poser $\log \widetilde{A}=2 S \log A$. Comme

$$
\left|\varphi\left(y_{j}\right)\right| \leq \mathrm{N}(\varphi) \sum_{i=1}^{d}\left|u_{i j}\right| \leq(1 / e) d D N \log A
$$

et que

$$
\left|s_{j}\right| \leq\left|\varphi\left(y_{j}\right)\right|+\frac{1}{2} \leq\left|\varphi\left(y_{j}\right)\right|+(1 /(4 e)) d D N \log A,
$$

on a $S \leq(1 / 2) d D N \log A$ et $D \log \widetilde{A} \leq d N(D \log A)^{2}$.

Si $d \geq 2$, alors le théorème 1.2 donne

$$
\max _{1 \leq j \leq \tilde{l}}\left|\varphi\left(\widetilde{y}_{j}\right)\right| \geq \mathrm{N}(\varphi) \exp \left\{-\widetilde{c}_{0}(D \log \widetilde{A})^{\tilde{\kappa}_{0}}\right\},
$$

avec $\widetilde{c}_{0}=c_{0}(d, \widetilde{l})$ et $\widetilde{\kappa}_{0}=\kappa_{0}(d, \widetilde{l})=\kappa_{1}$. Si $d=1$, on utilise l'inégalité de Liouville (4.1) à la place du théorème 1.2 :

$$
\max _{1 \leq j \leq \tilde{l}}\left|\varphi\left(\widetilde{y}_{j}\right)\right| \geq \mathrm{N}(\varphi) 2^{-D} \widetilde{A}^{-D} .
$$

Ceci démontre le corollaire 1.3.

5. Mesures de transcendance. Ce paragraphe est consacré à la démonstration du théorème 1.4 et à celle du corollaire 1.5. On utilisera de nouveau le théorème 2.1 de [24], mais avec $d_{0}=1$.

Démonstration du théorème 1.4. Pour éviter une confusion de notations entre le théorème 1.4 et le théorème 2.1 de [24], on va remplacer dans le théorème 1.4 l'entier $d$ par $d_{1}$. On suppose

$$
\max _{1 \leq j \leq l}\left|\varphi\left(y_{j}\right)-\beta_{j}\right|<\exp \left\{-c_{2}(D \log A)^{d_{1} \kappa_{2}}(D \log B)^{\kappa_{2}}\right\}
$$

et on veut en déduire une contradiction. Soit $S$ le plus petit entier satisfaisant

$$
S^{l-d_{1}^{2}}>4^{d_{1}+1}\left(d_{1}+1\right)\left(12 d_{1}+25\right)^{d_{1}\left(d_{1}+1\right)}\left(d_{1} l D \log A\right)^{d_{1}^{2}}(D \log B)^{d_{1}} .
$$

Alors $S$ vérifie

$$
(S / 2)^{l-d_{1}^{2}} \leq 4^{d_{1}+1}\left(d_{1}+1\right)\left(12 d_{1}+25\right)^{d_{1}\left(d_{1}+1\right)}\left(d_{1} l D \log A\right)^{d_{1}^{2}}(D \log B)^{d_{1}} .
$$


On définit deux nombres réels $U_{1}$ et $V_{1}$ par

$$
U_{1}=4\left(\left(12 d_{1}+25\right) d_{1} l S D \log A\right)^{d_{1}}(D \log B) \quad \text { et } \quad V_{1}=\left(12 d_{1}+25\right) U_{1} .
$$

En utilisant la majoration de $S$, on trouve

$$
\left(V_{1} / 2^{d_{1}}\right)^{l-d_{1}^{2}} \leq 4^{d_{1}+l}\left(d_{1}+1\right)^{d_{1}}\left(12 d_{1}+25\right)^{l\left(d_{1}+1\right)}\left(d_{1} l D \log A\right)^{d_{1} l}(D \log B)^{l} .
$$

Un petit calcul permet de vérifier que la constante

$$
c_{2}= \begin{cases}d_{1}^{17 d_{1}^{3}} l^{d_{1}} & \text { si } d_{1} \geq 2, \\ 10^{10}(l-1)^{2} & \text { si } d_{1}=1,\end{cases}
$$

du théorème 1.4 satisfait

$$
\left(\frac{c_{2}-\left(2 d_{1}\right)^{3} l}{2^{d_{1}}}\right)^{l-d_{1}^{2}}>4^{d_{1}+l}\left(d_{1}+1\right)^{d_{1}}\left(12 d_{1}+25\right)^{l\left(d_{1}+1\right)}\left(d_{1} l\right)^{d_{1} l} .
$$

En majorant $\log (l S)$ par $\left(2 d_{1}\right)^{3} l(D \log A)^{d_{1} \kappa_{2}}(D \log B)^{\kappa_{2}}$, on en déduit

$$
V_{1}+\log (l S) \leq c_{2}(D \log A)^{d_{1} \kappa_{2}}(D \log B)^{\kappa_{2}} .
$$

On va utiliser le théorème 2.1 de [24] avec

$$
\begin{gathered}
l_{0}=0, \quad d_{0}=1, \quad d=d_{1}+1, \quad l_{1}=l, \quad r=r_{3}=d_{1}, \quad r_{1}=r_{2}=0, \\
W=\mathcal{W}^{\prime}=\{0\}, \quad \log A_{i}=l S \log A, \quad E=e \quad \text { et } \quad M=S^{l} .
\end{gathered}
$$

On définit $A_{1}, \ldots, A_{d_{1}}, T_{0}, T_{1}, \ldots, T_{d_{1}}$ par

$$
\begin{gathered}
\log A_{i}=l S \log A \\
T_{i}=\left[U_{1} /\left(d_{1} D \log A_{i}\right)\right]=\left[U_{1} /\left(d_{1} l S D \log A\right)\right] \quad\left(1 \leq i \leq d_{1}\right)
\end{gathered}
$$

et

$$
T_{0}=\left[U_{1} /(D \log B)\right]
$$

Grâce aux choix de $S$ et de $U_{1}$, on vérifie sans difficulté l'hypothèse principale du $\S 2 \mathrm{~g}$ de [24], à savoir

$$
\left(T_{0}+1\right)\left(T_{1}+1\right) \ldots\left(T_{d_{1}}+1\right) \geq 4 V_{1}^{d_{1}} .
$$

On pose, pour $1 \leq j \leq l$,

$$
\eta_{j}=\left(\beta_{j}, u_{1 j}, \ldots, u_{d_{1} j}\right) \quad \text { et } \quad \eta_{j}^{\prime}=\left(\varphi\left(y_{j}\right), u_{1 j}, \ldots, u_{d_{1} j}\right) .
$$

Ainsi

$$
\left|\eta_{j}-\eta_{j}^{\prime}\right|=\left|\varphi\left(y_{j}\right)-\beta_{j}\right| \quad(1 \leq j \leq l) .
$$

On définit encore $2 M$ éléments de $\mathbb{C}^{d_{1}}$, indexés par $s=\left(s_{1}, \ldots, s_{l}\right) \in \mathbb{Z}^{l}$ avec $0 \leq s_{j}<S(1 \leq j \leq l)$, de la manière suivante :

$$
\eta_{s}=s_{1} \eta_{1}+\ldots+s_{l} \eta_{l} \quad \text { et } \quad \eta_{s}^{\prime}=s_{1} \eta_{1}^{\prime}+\ldots+s_{l} \eta_{l}^{\prime} .
$$

Ils sont reliés par

$$
\max _{s}\left|\eta_{s}-\eta_{s}^{\prime}\right| \leq l S \max _{1 \leq j \leq l}\left|\varphi\left(y_{j}\right)-\beta_{j}\right| \leq e^{-V_{1}} .
$$


Les points $\eta_{s}^{\prime}$ appartiennent à l'hyperplan $\mathcal{V}^{\prime}$ de $\mathbb{C}^{d_{1}+1}$ d'équation $z_{0}=$ $\varphi\left(z_{1}, \ldots, z_{d_{1}}\right)$. On désigne par $\Sigma$ le sous-ensemble suivant de $\mathbb{C} \times\left(\mathbb{C}^{\times}\right)^{d_{1}}$ :

$$
\begin{array}{r}
\Sigma=\left\{\left(s_{1} \beta_{1}+\ldots+s_{l} \beta_{l}, \alpha_{11}^{s_{1}} \ldots \alpha_{1 l}^{s_{l}}, \ldots, \alpha_{d_{1} 1}^{s_{1}} \ldots \alpha_{d_{1} l}^{s_{l}}\right) \in \mathbb{C} \times\left(\mathbb{C}^{\times}\right)^{d_{1}}:\right. \\
\left.s=\left(s_{1}, \ldots, s_{l}\right) \in \mathbb{Z}^{l}, 0 \leq s_{j}<S(1 \leq j \leq l)\right\} .
\end{array}
$$

On fait encore intervenir les deux projections $\Sigma_{0}$ et $\Sigma_{1}$ de $\Sigma$ sur chacun des deux facteurs :

$\Sigma_{0}=\left\{s_{1} \beta_{1}+\ldots+s_{l} \beta_{l} \in \mathbb{C}: s=\left(s_{1}, \ldots, s_{l}\right) \in \mathbb{Z}^{l}, 0 \leq s_{j}<S(1 \leq j \leq l)\right\}$

et

$$
\begin{aligned}
& \Sigma_{1}=\left\{\left(\alpha_{11}^{s_{1}} \ldots \alpha_{1 l}^{s_{l}}, \ldots, \alpha_{d_{1} 1}^{s_{1}} \ldots \alpha_{d_{1} l}^{s_{l}}\right) \in\left(\mathbb{C}^{\times}\right)^{d_{1}}:\right. \\
& \left.\quad s=\left(s_{1}, \ldots, s_{l}\right) \in \mathbb{Z}^{l}, 0 \leq s_{j}<S(1 \leq j \leq l)\right\} .
\end{aligned}
$$

Les conditions pour appliquer le théorème 2.1 de [24] sont toutes remplies. On en déduit l'existence d'un sous-groupe algébrique connexe $G^{*}=G_{0}^{*} \times G_{1}^{*}$ de $G=\mathbb{G}_{\mathrm{a}} \times \mathbb{G}_{\mathrm{m}}^{d_{1}}$, distinct de $G$, tel que, si on pose

$d_{0}^{*}=\operatorname{dim}\left(\mathbb{G}_{\mathrm{a}} / G_{0}^{*}\right), \quad d_{1}^{*}=\operatorname{dim}\left(\mathbb{G}_{\mathrm{m}}^{d_{1}} / G_{1}^{*}\right) \quad$ et $\quad \Sigma^{*}=\left(\Sigma+G^{*}(K)\right) / G^{*}(K)$, on ait

$$
\text { Card } \Sigma^{*} \leq\left(d_{1}+1\right) T_{0}^{d_{0}^{*}} T_{1}^{d_{1}^{*}} .
$$

On distingue maintenant deux cas.

(a) Supposons $d_{1}^{*} \geq 1$, c'est-à-dire $G_{1}^{*} \neq G_{1}$. Posons

$$
\Sigma_{1}^{*}=\left(\Sigma_{1}+G_{1}^{*}(K)\right) / G_{1}^{*}(K) .
$$

L'injection de $G_{1} / G_{1}^{*}$ dans $G / G^{*}$ montre que l'on a Card $\Sigma_{1}^{*} \leq$ Card $\Sigma^{*}$. Or les paramètres ont été choisis de telle sorte que

$$
S^{l}>\left(d_{1}+1\right) T_{0} T_{1}^{d_{1}} .
$$

Ainsi on déduit de (5.1)

$$
\operatorname{Card} \Sigma_{1}^{*} \leq \operatorname{Card} \Sigma^{*}<S^{l} .
$$

Alors on trouve d'abord $d_{1} \geq 2$, puis, en utilisant le lemme $4.2, d_{1}^{*}=1$ et Card $\Sigma_{1}^{*} \geq S^{l-1}$. Grâce à (5.1) on obtient

$$
S^{l-1} \leq\left(d_{1}+1\right) T_{0} T_{1}
$$

Mais, comme $d_{1} \geq 2$, on a $U_{1} \geq d_{1} l S^{2} D \log A$, donc $T_{1} \geq S$, ce qui montre que cette majoration (5.3) n'est pas compatible avec la minoration (5.2).

(b) Supposons maintenant $d_{1}^{*}=0$, c'est-à-dire $G_{1}^{*}=G_{1}$. Alors $d_{0}^{*}=1$ (puisque $G^{*}$ est distinct de $G$ ). On déduit de (5.1)

$$
\text { Card } \Sigma_{0} \leq\left(d_{1}+1\right) T_{0} \text {. }
$$

Il en résulte que l'ensemble

$$
\mathcal{E}_{1}=\left\{\left(s_{1}, \ldots, s_{l}\right) \in \mathbb{Z}^{l}:\left|s_{j}\right|<S(1 \leq j \leq l), s_{1} \beta_{1}+\ldots+s_{l} \beta_{l}=0\right\}
$$


satisfait Card $\mathcal{E}_{1} \geq S^{l} /\left(\left(d_{1}+1\right) T_{0}\right)$. Nous allons utiliser le théorème 4.3 afin de majorer Card $\mathcal{E}_{1}$. Pour $s \in \mathcal{E}_{1}$ on a

$$
\left|\varphi\left(s_{1} y_{1}+\ldots+s_{l} y_{l}\right)\right| \leq l S \max _{1 \leq j \leq l}\left|\varphi\left(y_{j}\right)-\beta_{j}\right| \leq e^{-V_{1}} .
$$

On a supposé $\left(\beta_{1}, \ldots, \beta_{l}\right) \neq(0, \ldots, 0)$. De l'inégalité de Liouville on tire

$$
B^{-D} \leq \max _{1 \leq j \leq l}\left|\beta_{j}\right| \leq \max _{1 \leq j \leq l}\left|\varphi\left(y_{j}\right)-\beta_{j}\right|+\frac{1}{e} \mathrm{~N}(\varphi) D \log A .
$$

Alors en utilisant la majoration $(l S)^{-1} e^{-V_{1}} \leq(1 / 2) B^{-D}$, on trouve

$$
\mathrm{N}(\varphi) \geq B^{-D}(D \log A)^{-1} .
$$

Le paramètre $V$ du théorème 4.3 est majoré par $V_{1}-D \log B-\log (D \log A)$, donc $e^{-V_{1}} \leq \mathrm{N}(\varphi) e^{-V}$, et l'ensemble $\mathcal{E}_{1}$ est contenu dans l'ensemble $\mathcal{E}$ du théorème 4.3 :

$$
\operatorname{Card} \mathcal{E}_{1} \leq \operatorname{Card} \mathcal{E} \leq 4^{d_{1}}\left(\left(12 d_{1}+13\right) d_{1} l S D \log A\right)^{d_{1}\left(d_{1}-1\right)} .
$$

L'estimation

$$
S^{l} /\left(\left(d_{1}+1\right) T_{0}\right) \leq 4^{d_{1}}\left(\left(12 d_{1}+13\right) d_{1} l S D \log A\right)^{d_{1}\left(d_{1}-1\right)}
$$

qui en résulte n'est pas compatible avec le choix de $S$. Ceci conclut la démonstration du théorème 1.4 .

Démonstration du corollaire 1.5. On applique le théorème 1.4 avec $d=1$ et $l=2$, ce qui donne $\kappa_{2}=2$ et $c_{2}=10^{10}$. On définit $\beta_{1}=1$, $\beta_{2}=\beta$ et $\varphi(z)=z / \lambda_{1}$, de sorte que $\varphi\left(\lambda_{2}\right)=\lambda_{2} / \lambda_{1}$ et $\mathrm{N}(\varphi)=1 /\left|\lambda_{1}\right|$. Le corollaire 1.5 en résulte.

6. Mesures de densité. On combine les théorèmes 1.2 et 1.4 avec la proposition 3.7 pour obtenir un énoncé de densité dans $\mathbb{R}^{r_{1}} \times \mathbb{C}^{r_{2}}$ d'un sous-groupe de type fini dont les éléments ont pour coordonnées des logarithmes de nombres algébriques. On obtiendra le théorème 1.6 en utilisant l'application exponentielle.

Proposition 6.1. Soient $r_{1}$ et $r_{2}$ des entiers $\geq 0$ avec $n=r_{1}+r_{2}>0$. On pose aussi $d=r_{1}+2 r_{2}$. Soit $Y$ un sous-groupe de type fini de $\mathcal{L}^{n}$ contenu dans $\mathbb{R}^{r_{1}} \times \mathbb{C}^{r_{2}}$. On choisit une base $y_{1}, \ldots, y_{l}$ de $Y$ sur $\mathbb{Z}$ et on écrit $y_{j}=$ $\left(u_{1 j}, \ldots, u_{n j}\right)(1 \leq j \leq l)$, avec $u_{i j} \in \mathcal{L} \cap \mathbb{R}$ pour $1 \leq i \leq r_{1}$ et $u_{i j} \in \mathcal{L} \subset \mathbb{C}$ pour $r_{1}+1 \leq i \leq n$. On suppose que pour tout $a=\left(a_{1}, \ldots, a_{d}\right) \in \mathbb{Z}^{d}, a \neq 0$, et tout $b=\left(b_{1}, \ldots, b_{l}\right) \in \mathbb{Z}^{l}, b \neq 0$, on $a$

$$
\sum_{j=1}^{l} b_{j}\left(\sum_{i=1}^{n} a_{i} u_{i j}+\sum_{k=r_{1}+1}^{n} a_{n+k} \bar{u}_{k j}\right) \neq 0 .
$$


(a) On suppose $l \geq \max \left\{2, d^{2}-d+1\right\}$ et on pose

$$
\theta_{1}=\frac{1}{d}-\frac{d-1}{l-1} .
$$

Il existe deux constantes positives $c_{3}^{\prime}$ et $c_{4}^{\prime}$, ne dépendant que de $d, l$ et des $u_{i j}$, possédant la propriété suivante : pour tout $\xi=\left(\xi_{1}, \ldots, \xi_{n}\right) \in \mathbb{R}^{r_{1}} \times \mathbb{C}^{r_{2}}$, et pour tout $T \geq c_{3}^{\prime} \max _{1 \leq i \leq n}\left(1+\left|\xi_{i}\right|\right)$, le système d'inéquations

$$
\max _{1 \leq j \leq l}\left|t_{j}\right| \leq T \quad \text { et } \max _{1 \leq i \leq n}\left|\xi_{i}-t_{1} u_{i 1}-\ldots-t_{l} u_{i l}\right| \leq c_{4}^{\prime}(\log T)^{-\theta_{1}}
$$

admet une solution $\left(t_{1}, \ldots, t_{l}\right) \in \mathbb{Z}^{l}$.

(b) On suppose $l>d^{2}$ et on définit

$$
\theta_{2}= \begin{cases}\left(l-d^{2}\right) / l & \text { si } d \geq 2, \\ 1 & \text { si } d=1 .\end{cases}
$$

Alors la même conclusion vaut avec la borne

$$
\max _{1 \leq i \leq n}\left|\xi_{i}-t_{1} u_{i 1}-\ldots-t_{l} u_{i l}\right| \leq \exp \left\{-c_{5}^{\prime}(\log T)^{\theta_{2}}\right\},
$$

où la constante $c_{5}^{\prime}$ ne dépend que de $d, l$ et des $u_{i j}$.

Démonstration. Pour $1 \leq j \leq l$ et $n<i \leq d$, on définit $u_{i j}=\bar{u}_{i-n, j}$. On définit ensuite $\widetilde{y}_{j} \in \mathbb{C}^{d}$ par $\widetilde{y}_{j}=\left(u_{1 j}, \ldots, u_{d j}\right)(1 \leq j \leq l)$. Alors $\widetilde{Y}=\mathbb{Z} \widetilde{y}_{1}+\ldots+\mathbb{Z} \widetilde{y}_{l}$ possède la propriété (IL).

Si $\theta_{1}=0$, c'est-à-dire $l=d^{2}-d+1$, on utilise la partie (a) du théorème 1.1 pour montrer que $\tilde{Y}$ n'est pas contenu dans un hyperplan complexe de $\mathbb{C}^{d}$, ce qui entraîne que $Y$ n'est pas contenu dans un hyperplan réel de $\mathbb{R}^{r_{1}} \times \mathbb{C}^{r_{2}}$. Alors $\left\{y_{1}, \ldots, y_{l}\right\}$ contient une base du $\mathbb{R}$-espace vectoriel $\mathbb{R}^{r_{1}} \times \mathbb{C}^{r_{2}}$. La proposition 6.1 ne dit rien de plus dans ce cas.

On suppose maintenant $l \geq d^{2}-d+2$. Pour démontrer la partie (a) de la proposition 6.1 , on applique le corollaire 1.3 afin de vérifier l'hypothèse de la proposition 3.7 avec

$$
F(S)=\exp \left(c_{13} S^{\kappa_{1}}\right) \quad \text { et } \quad \theta_{1}=\frac{1}{\kappa_{1}}=\frac{1}{d}-\frac{d-1}{l-1} .
$$

Pour conclure, on minore $G$ par $G(T) \geq\left(1 / c_{4}^{\prime}\right)(\log T)^{\theta_{1}}$.

Supposons ensuite $l \geq d^{2}+1$ et $d \geq 2$. Pour $1 \leq j \leq l$, soit $\beta_{j} \in \mathbb{Z}$ un nombre entier à distance minimale de $\varphi\left(y_{j}\right)$. Si $\left(\beta_{1}, \ldots, \beta_{l}\right)=(0, \ldots, 0)$, on applique le théorème 1.2. Sinon, c'est le théorème 1.4 que l'on utilise. Dans les deux cas on vérifie les hypothèses de la proposition 3.7 avec

$$
F(S)=\exp \left(c_{14}(\log S)^{\kappa_{2}}\right) \quad \text { et } \quad \theta_{2}=\frac{1}{\kappa_{2}}=1-\frac{d^{2}}{l} .
$$

Enfin, quand $d=1$, on a $r_{1}=n=1, r_{2}=0$ et $l \geq 2$. Le résultat que nous voulons démontrer s'énonce alors de la façon suivante : 
Soient $\alpha_{1}$ et $\alpha_{2}$ deux nombres algébriques réels positifs multiplicativement indépendants. Il existe deux constantes positives $c_{3}^{\prime}$ et $c_{5}^{\prime}$, ne dépendant que de $\alpha_{1}$ et $\alpha_{2}$, telles que, pour tout nombre réel $x$ et tout $T \geq c_{3}^{\prime}(1+|x|)$, le système d'inéquations

$$
\max \left\{\left|t_{1}\right|,\left|t_{2}\right|\right\} \leq T \quad \text { et } \quad\left|x-t_{1} \log \alpha_{1}-t_{2} \log \alpha_{2}\right| \leq T^{-c_{5}^{\prime}}
$$

admet une solution $\left(t_{1}, t_{2}\right) \in \mathbb{Z}^{2}$.

On déduit cet énoncé du lemme 3.5 , grâce à la mesure d'irrationalité suivante, due à Fel'dman [5], chap. 10, th. 3.10 : il existe une constante $c_{15}>0$, ne dépendant que de $\alpha_{1}$ et $\alpha_{2}$, telle que, pour tout $p / q \in \mathbb{Q}$ avec $q>0$, on ait

$$
\left|\frac{\log \alpha_{1}}{\log \alpha_{2}}-\frac{p}{q}\right|>q^{-c_{15}}
$$

Cela permet d'utiliser le lemme 3.5 (ou bien la proposition 3.7, au choix) avec $F(S)=S^{c_{16}}$ et $G(T) \geq T^{c_{5}^{\prime}}$.

Démonstration du théorème 1.6. Pour $1 \leq j \leq l$, on pose

$$
u_{i j}=\log \alpha_{i j} \quad\left(1 \leq i \leq r_{1}\right),
$$

où log est la détermination réelle du logarithme, puis on choisit $u_{r_{1}+1, j}, \ldots$ $\ldots, u_{n j}$ dans $\mathbb{C}$ tels que $\exp \left(u_{i j}\right)=\alpha_{i j}$ pour $r_{1}<i \leq n$ et on définit $y_{j}=\left(u_{1 j}, \ldots, u_{n j}\right) \in \mathbb{R}^{r_{1}} \times \mathbb{C}^{r_{2}}(1 \leq j \leq l)$.

Soit $\zeta \in\left(\mathbb{R}_{+}^{\times}\right)^{r_{1}} \times\left(\mathbb{C}^{\times}\right)^{r_{2}}$. On pose $\xi_{i}=\log \zeta_{i}$ pour $1 \leq i \leq r_{1}$. Ensuite, pour $r_{1}<i \leq n$ on définit $\xi_{r_{1}+i}$ comme la détermination principale du logarithme de $\zeta_{r_{1}+i}$. Ainsi on a, pour $1 \leq i \leq n$,

$$
\left|\xi_{i}\right|^{2} \leq\left(\log \left|\zeta_{i}\right|\right)^{2}+\pi^{2} \leq\left(\pi^{2}+1\right) \max \left\{1,\left(\log \left|\zeta_{i}\right|\right)^{2}\right\}
$$

et

$$
1+\left|\xi_{i}\right| \leq 5 \max \left\{1,|\log | \zeta_{i}||\right\}
$$

Le théorème 1.6 résulte alors de la proposition 6.1 .

\section{Références}

[1] A. Baker, Transcendental Number Theory, 2nd ed., Cambridge Univ. Press, 1979.

[2] N. Bourbaki, Eléments de mathématiques, Livre III : Topologie générale, Chapitre 5 : Groupes à un paramètre, Chapitre 7 : Les groupes additifs $\mathbb{R}^{n}$, Hermann, Paris, 1974.

[3] J. W. S. Cassels, An Introduction to Diophantine Approximation, Cambridge Tracts in Math. and Math. Phys. 45, Cambridge Univ. Press, 1957.

[4] M. Emsalem, Sur les idéaux dont l'image par l'application d'Artin dans une $\mathbb{Z}_{p}$-extension est triviale, J. Reine Angew. Math. 382 (1987), 181-198.

[5] N. I. Fel'dman, Hilbert's Seventh Problem, Moscow State University, Moscow, 1982, 311 pp. 
[6] A. O. Gel'fond, Transcendental Number Theory, Moscow, 1952 (en russe); trad. angl., Dover, New York, 1960.

[7] G. H. Hardy and E. M. Wright, An Introduction to the Theory of Numbers, 5th ed., Oxford Science Publ., 1979.

[8] S. Lang, Introduction to Transcendental Numbers, Addison-Wesley, 1966.

[9] M. Laurent, M. Mignotte et Yu. V. Nesterenko, Formes linéaires en deux logarithmes et déterminants d'interpolation, J. Number Theory 55 (1995), 285-321.

[10] K. Ramachandra, Contributions to the theory of transcendental numbers $(I)$, Acta Arith. 14 (1968), 65-72; (II), ibid., 73-88.

[11] D. Roy, Sur une version algébrique de la notion de sous-groupe minimal relatif de $\mathbb{R}^{n}$, Bull. Soc. Math. France 118 (1990), 171-191.

[12] -, Sous-groupes minimaux des groupes de Lie commutatifs réels et applications arithmétiques, Acta Arith. 56 (1990), 257-269.

[13] - , Transcendance et questions de répartition dans les groupes algébriques, dans : Approximations Diophantiennes et Nombres Transcendants, Luminy 1990, P. Philippon (éd.), de Gruyter, 1992, 249-274.

[14] —, Simultaneous approximation in number fields, Invent. Math. 109 (1992), 547556.

[15] J.-J. Sansuc, Descente et principe de Hasse pour certaines variétés rationnelles, dans : Séminaire de Théorie des Nombres de Paris 1980-81, M. J. Bertin (éd.), Progr. Math. 22, Birkhäuser, 1982, 253-271.

[16] W. M. Schmidt, Diophantine Approximation, Lecture Notes in Math. 785, Springer, 1980; 2nd printing, 1996.

[17] T. Schneider, Einführung in die transzendenten Zahlen, Springer, 1957; trad. franç., Introduction aux Nombres Transcendants, Gauthier-Villars, Paris, 1959.

[18] M. Waldschmidt, Propriétés arithmétiques de fonctions de plusieurs variables (III), dans : Sém. P. Lelong-H. Skoda (Analyse), 19ème année, 1978/79; Lecture Notes in Math. 822, Springer, 1980, 332-356.

[19] —, Nombres transcendants et groupes algébriques, Astérisque 69/70 (1979).

[20] —, Dependence of logarithms of algebraic points, dans : Colloq. Math. Soc. János Bolyai 51, North-Holland, 1987, 1013-1035.

[21] - On the transcendence methods of Gel'fond and Schneider in several variables, dans : New Advances in Transcendence Theory, Proc. Conf. Durham 1986, A. Baker (ed.), Cambridge Univ. Press, 1988, 375-398.

[22] - Densité de points rationnels sur un groupe algébrique, Experiment. Math. 3 (1994), 329-352 et 4 (1995), 255.

[23] - Topologie des points rationnels, Cours de Troisième Cycle 1994/95, Université P. et M. Curie (Paris VI); http://www.math.jussieu.fr/ miw

[24] - Approximation diophantienne dans les groupes algébriques commutatifs, soumis pour publication.

Institut Mathématique de Jussieu

Problèmes Diophantiens, Case 247

Université P. et M. Curie (Paris VI)

4, Place Jussieu

F-75252 Paris Cedex 05, France

E-mail: miw@math.jussieu.fr 